Published in final edited form as:

Nature. 2018 November 1; 563(7730): 275-279. doi:10.1038/s41586-018-0672-3.

\title{
Conformational transitions of the serotonin 5-HT3 receptor
}

\author{
Lucie Polovinkin ${ }^{1}$, Ghérici Hassaine ${ }^{2}$, Jonathan Perot ${ }^{1}$, Emmanuelle Neumann ${ }^{1}$, Anders A. \\ Jensen $^{3}$, Solène Lefebvre ${ }^{4}$, Pierre-Jean Corringer ${ }^{4}$, Jacques Neyton ${ }^{1}$, Christophe \\ Chipot $^{5,6,7}$, Francois Dehez ${ }^{5,6}$, Guy Schoehn ${ }^{1}$, and Hugues Nury ${ }^{1}$ \\ ${ }^{1}$ CNRS, Univ. Grenoble Alpes, CEA, IBS, Grenoble, France \\ ${ }^{2}$ Theranyx, Marseille, France \\ ${ }^{3}$ Department of Drug Design and Pharmacology, Faculty of Health and Medical Sciences, \\ University of Copenhagen, Denmark \\ ${ }^{4}$ Channel Receptors Unit, CNRS UMR 3571, Institut Pasteur, Paris, France \\ ${ }^{5}$ Université de Lorraine, CNRS, LPCT, F-54000 Nancy, France \\ ${ }^{6}$ Laboratoire International Associé CNRS and University of Illinois at Urbana-Champaign, \\ Vandoeuvre-les-Nancy, France \\ ${ }^{7}$ Department of Physics, University of Illinois at Urbana-Champaign, Urbana, Illinois, USA
}

\section{Abstract}

The serotonin 5-HT3 receptor is a pentameric ligand-gated ion channel (pLGIC). It belongs to a large family of receptors that function as allosteric signal transducers across the plasma membrane ${ }^{1,2}$ : upon binding of neurotransmitter molecules to extracellular sites, the receptors undergo complex conformational transitions, which result in transient opening of a pore permeable to ions. 5-HT3 receptors are therapeutic targets for emesis and nausea, irritable bowel syndrome and depression ${ }^{3}$. Despite the recent accumulation in pLGIC structures ${ }^{4-8}$, no clear unifying view has emerged on conformational transitions involved in channel gating. Here we report four cryoEM structures of the full-length mouse 5-HT3 receptor, ranging from $3.2 \AA$ to $4.5 \AA$ resolution, obtained in complex with the anti-emetic drug tropisetron, with serotonin, with serotonin and a positive allosteric modulator. The tropisetron-bound structure resembles those obtained with an inhibitory nanobody ${ }^{5}$ or without ligand ${ }^{9}$. The other structures represent new conformations, including that of an open state and of two novel ligand-bound states. We present computational insights into the dynamics of the structures, their pore hydration and free-energy profiles; we

\footnotetext{
Correspondence and requests for materials should be addressed to H.N (hugues.nury@ibs.fr) or J.N (jacques.neyton@ibs.fr). Data availability. Atomic coordinates of the four conformations have been deposited in the Protein Data Bank with accession numbers $6 \mathrm{HIN}, 6 \mathrm{HIO}, 6 \mathrm{HIQ}$ and $6 \mathrm{HIS}$ for conformations F, I1, I2 and T, respectively. The cryo-EM density maps have been deposited in the Electron Microscopy Data Bank with accession numbers EMD-0225, EMD-0226, EMD-0227 and EMD-0228 for conformations F, I1, I2 and T, respectively.

Author contributions

L.P., J.P. and G.H performed sample preparation. L.P., H.N, E.N. and G.S optimized and collected microscopy data. L.P and H.N built the models. F.D and C.C. conducted MD simulations. J.N. performed electrophysiology experiments. S.L and P.J.C performed VCF experiments. A.J performed FLIPR experiments. All authors extensively discussed the data, H.N and J.N wrote the manuscript with inputs from all authors.

Competing interest. G.H is employed by Theranyx
} 
characterize motions at the gate level and cation accessibility in the pore. Put together, the data deepen our understanding of the gating mechanism of pLGICs, while capturing ligand binding in unprecedented detail.

A decade after the seminal structure of the Torpedo nicotinic acetylcholine receptor ${ }^{10}$ (nAChR), the set of known pLGIC structures is under rapid expansion and reflects the diversity of the family. These structures all share a conserved architecture, where subunits are arranged around a central 5-fold pseudo-symmetry axis. Together they have unveiled the detail of ligand binding, selectivity and allosteric modulation. They have also revealed a complex landscape of conformations, raising questions on how to relate structures to the wealth of data that established the existence of several agonist-bound pre-active intermediate states $^{11-13}$, of distinct open states ${ }^{14}$, and of multiple desensitized states ${ }^{15}$.

Mouse homomeric 5-HT3A receptors, with their entire intracellular domain (ICD), were solubilized with the detergent C12E9 and purified. We first performed cryo-electron microscopy (cryo-EM) in the presence of the potent antagonist tropisetron and obtained a 4.5 ̊ structure (Fig. 1b, Extended Data Fig. 1-3) referred to as T hereafter. T is globally similar to the structure previously solved by X-ray crystallography ${ }^{5}$ (RMSD of $0.6 \AA$ ), the pore of which was shown by molecular dynamics (MD) to be occluded ${ }^{16}$. Tropisetron fits in a peanut-shaped density present in the neurotransmitter pocket (Extended Data Fig 4d-f, 6f). The ICD contains a zone of about 60 residues, which is averaged out (also in the other reconstructions, see below) because of its intrinsic flexibility ${ }^{1,2}$. T resembles the $4.5 \AA$ cryoEM structure of the apo 5-HT3 receptor ${ }^{9}$ (RMSD of $1.15 \AA$ ), with differences in the lipidexposed helices M3, Mx and M4.

We then sought to identify agonist-elicited conformations of the 5-HT3 receptor and performed cryo-EM imaging in the presence of serotonin. A first reconstruction presented heterogeneity in the membrane domain. Further focused 3D classification allowed two subsets of particles to be separated, which yielded reconstructions at $4.2 \AA$ and $4.1 \AA$ resolution, corresponding to two different conformations (Fig. 1b, Extended Data Fig. 5). The maps offer a variable level of information: most side chains are resolved in the extracellular domain, whereas some parts of the membrane domains do not have side-chain information, and some even have limited information in the main chain position (Extended Data Fig. 5c, 6), reflecting the receptor dynamics. In the two refined structures, the extracellular domains (ECD) have undergone an equivalent transition from the $\mathrm{T}$ state and serotonin could be modelled in the neurotransmitter site, while transmembrane domains (TMD) differed markedly (Fig. 2, Extended Data Fig. 7). We call the first structure I1 for intermediate 1 and the second structure $\mathrm{F}$ for full, based on the extent of motions compared to the inhibited state. I1 exhibits only limited motions in the upper part of M1 and M2, and a rearrangement of the M2-M3 loop (Video S1). F is characterized by a pronounced reorganisation of the transmembrane helices, which can be described by a rigid-body motion of the four-helix bundle coupled to a rearrangement of M4 (and of M3 to a lower extend) sliding along M1 and M2 (Videos S2 and S3). It also features a very dynamic ICD, beyond the intrinsically disordered region, where model building was not possible even though the data showed incomplete densities for Mx and M4 (Extended Data Fig. 5a-c). 
We finally collected a dataset in the presence of $30 \mu \mathrm{M}$ serotonin and $100 \mu \mathrm{M}$ trans-3-(4methoxyphenyl)-N-(pentan-3-yl)acrylamide (TMPPAA, a compound showing agonist and positive allosteric modulator properties on the human receptor ${ }^{17}$ ), a combination that yields weakly desensitizing currents (Extended Data Fig. 1f). The dataset allowed a $3.2 \AA$ resolution reconstruction (Extended Dat Fig. 3d-g), which provides non-ambiguous sidechain information throughout nearly the entire receptor. The refined structure has an ECD conformation essentially equivalent to that of I1 and F. The membrane domain is close to that of I1, albeit slightly more expanded on the top part and in the pore (Fig. 2). We call this structure $\mathrm{I} 2$ for intermediate 2 .

Serotonin can be unambiguously positioned in the neurotransmitter site of I2. It fits tightly within its binding pocket (Extended Data Fig. 4a-c) in an orientation that recapitulates the wealth of knowledge gained from functional and binding studies ${ }^{1}$. Surrounded by obligatory aromatic residues (F199 and Y207 on the principal side, Y126 and W63 on the complementary side), it is positioned to form a cation- $\pi$ interaction with W156 and hydrogen bonds with the main chain of S155 and Y64. The C-loop underwent a moderate inward motion compared to the inhibited conformations, its position is locked by a salt bridge between D202 and R65. A hallmark of allosteric activation is the subunit/subunit rearrangement, which impacts the site volume and geometry.

TMPPAA has been proposed to bind to an allosteric site in the $\mathrm{TMD}^{17}$, but no clearly interpretable density is found in our data to model the compound. We tested the TMPPAA agonist activity on a set of $\sim 45$ single-point mutants of the human receptor, which collectively reveal that the drug binds between M4, M1 and M3, into an intra-subunit cavity skirted by lipids of the outer leaflet in the upper part of the TMD (Extended Data Fig. 8), where endogenous steroids bind to nAChRs ${ }^{18}$. More generally, several allosteric druggable sites have been identified in pLGICs, wherein diverse compounds bind, ranging from general anesthetics like propofol to flavorings like citral or eucalyptol ${ }^{3}$. Allosteric sites in the 5-HT3 receptor change in both shape and volume during transitions (Extended Data Fig. $4 \mathrm{i}, 8 \mathrm{a})$.

At the ECD-TMD interface, a set of conserved residues essential for gating ${ }^{19}$ form a striking structural motif ubiquitous to all pLGIC structures, the location of which may correlate with the state of the channel ${ }^{5}$. This motif consists of charged residues (E53, D145, E186, R218), sandwiched between conserved aromatic ones: W187 at the top and the 142-FPF-144 motif of the cys-loop, plus Y223 at the bottom (Extended Data Fig. 9). The FPF motif itself penetrates the transmembrane domain like a wedge; its position differs in each conformations. When superimposing structures on a TMD subunit, the wedge lies close to $\mathrm{M} 2$ in the tropisetron-bound structure and moves toward M4-M1 in I1, I2 and F structures (which is possible because the conserved P230 allows the upper M1 to kink or straighten). A marked downward concerted movement of the wedge and of the $\beta 8$ - $\beta 9$ loop (containing E186 and W187) occurs in the F structure, pushing on the M2-M3 loop, and may be responsible for the important TMD reorganisation observed in that state.

pLGIC pores are lined by side chains of residues from the five M2 helices. In direct agreement with substituted cysteine accessibility mutagenesis (SCAM, reviewed by 
Thompson et al. ${ }^{1}$ ), our structures show that, in all conformations, positions -1', 2', 6', 9', 13', 16', 17', 20' are exposed to the pore lumen (Fig. 3a). Positions 12' and 15', also found to be accessible by SCAM, are partly solvent-exposed on the back side of M2s.

Superimposition of a single M2 underscores its flexibility at both ends; superimposition of the five M2s highlights the crucial role of motions of the hydrophobic side chain at 9' (Fig. 3b-c). Minimal pore diameter is often a cornerstone in the assignment of experimental structures to physiological states. Pore profiles are compatible with closed hydrophobic gates (rings at positions 9',13', 16' and 17') for the T and I1 structures, with an open channel for the F structure, while I2 presents an intermediate profile. However, pore profiles are influenced by the resolution, the symmetry, and by the rotameric state of those side chains pointing to the pore lumen. Some positions such as the key negative charge in -1' are often poorly resolved in density maps and known to adopt alternate conformations ${ }^{8,20}$. Moreover, pore profiles are not informative about hydrophobicity, wetting or dynamics, which play key roles in permeability ${ }^{21,22}$. We performed MD simulations to better characterize permeation. In the $\mu$ s-long trajectories starting from the inhibited X-ray structure, or from I1, no water or ions cross the pore, and the hydrophobic 9', 13' and 16' rings establish a dewetted hydrophobic gate tighter than in the starting structures (Fig. 3d, Extended Data Fig. 10). In sharp contrast, a simulation starting from $\mathrm{F}$ features an open pore accessible to ions and water throughout the trajectory (Video S4). During the initial part of the simulations starting from I2, when the $\mathrm{C} a$ positions are positionally restrained, wetting and dewetting events of the pore occur as if its conformation were on the open-closed verge (Extended Data Fig. 10). Wetting is linked to the presence of transiently hydrated grooves at the back of M2s, down to the polar residues Y11'-S12', affecting the electrostatic landscape inside the pore. Wetting also correlates with rotation of L260 out of the pore lumen. Once the geometric restraints are removed, the I2 pore relaxes to a closed conformation similar to that observed in the I1 trajectory. The absence of TMPPAA in the simulation may rationalize the closure. Potentials of mean force for the translocation of a $\mathrm{K}^{+}$ion reveal an insuperable $12-\mathrm{kcal} / \mathrm{mol}$ barrier in the case of I1 (representative of closed hydrophobic gate conformations) and an essentially flat free-energy landscape in the case of F (Fig. 3e).

Can the structures be assigned to physiological states? While $\mathrm{T}$ and $\mathrm{F}$ are straightforward to assign, I1 and I2 less so. T typifies an inhibited state (resting-like ECD stabilized by tropisetron, closed pore) resembling the apo state. F represents an open state (activated ECD with bound serotonin, activated TMD and widely open pore). Two possible assignments can be proposed for the closely related I1 and I2 conformations (Fig 4a). In a first scheme, I1 exemplifies a serotonin-bound pre-active closed state, where the ECD and the ECD/TMD interface, but not yet the TMD, have undergone a transition. Indeed, 5-HT3 receptor singlechannel analysis yields kinetic models where opening can happen from a ligand-bound preactive state ${ }^{14}$. In a second scheme, I1 represents a closed desensitized state that occurs downstream from the open state. In either scheme, I 2 is best described as a state close to I1 (i.e either close to pre-active or desensitized), wherein the slightly wider pore promotes wetting, which could enable ion passage or merely favor the switch to a fully open state, in line with the TMPPAA-induced modulation.

A distinctive feature of the second scheme is that the activation gate, consisting of rings of hydrophobic residues in the upper pore, would open in the active state and then close in the 
desensitized state. It implies a marked movement of the upper pore during desensitization, and no ion access from the extracellular compartment to the lower pore in the desensitized state. Functional work in anionic receptors points to distinct activation and desensitization gates, the latter being located at the cytoplasmic end of the pore ${ }^{23}$. Moreover structures of the GABA $\beta 3$ and of the nACh $a 4 \beta 2$ receptors, with pores constricted at positions -2' and $-1^{\prime}$, have been assigned to desensitized states ${ }^{6,8}$. These receptors, however, were engineered close to the constriction, a feature that can alter desensitization in 5-HT3 receptors ${ }^{24}$.

To challenge the two schemes we measured the motion of the gate region, as well as the accessibility of the pore to organic cations. First, we used voltage-clamp fluorometry (VCF) to probe the local conformational changes in the upper pore at position 19,25,26 (Extended Data Fig. 11). S19'C mutants expressed at the surface of Xenopus oocytes were labeled with MTS-TAMRA. Short serotonin applications elicited simultaneous current and fluorescence changes with parallel rise and decay, while prolonged 7-minute applications of serotonin resulted in similar signals at the onset, followed by a slow decay for the current signal bereft of change in fluorescence. Both signals returned to baseline when serotonin was removed. The VCF data rather argue against the second scheme since the probe environment changes upon activation but not during desensitization. Second, we performed SCAM in the resting (absence of ligand), open (during short serotonin application) and desensitized (after prolonged serotonin application) states (Fig. 4b-c). MTSEA and MTSET are organic cations reacting with free cysteines through their methanethiosulfonate moiety. The aminoethyl head group of MTSEA is small, which can enable an enhanced access to narrow spaces. MTSEA is, however, also known to cross membranes in its uncharged form. Application of $1 \mathrm{mM}$ extracellular MTSEA in the resting state yielded no modification of currents in T6' $\mathrm{C}$ and S2'C mutants but inhibited currents in E-1'C (32\%). In that state, the compound can probably access position -1' from the internal compartment, but it can access neither 2' nor 6'. Similar applications in the open state produced irreversible inhibitions at positions 6', 2' and -1 ' (82\%, 69\% and 58\% respectively). MTSEA can therefore reach 6' and 2' from the extracellular compartment in the open state, in line with previous studies ${ }^{1}$ and consistent with the opening of the activation gate in F. Applications of extracellular MTSEA in the desensitized state also produced irreversible inhibitions at 6', 2' and $-1^{\prime}$ ' $(30 \%, 63 \%$ and $50 \%$ respectively). We infer that positions 6' and 2', located below the hydrophobic gate seen in T and I1, are accessible from the outside in the desensitized state. No conclusions can be drawn for position -1 ', its labeling in the resting state precluding further interpretation. The results suggest that the activation gate is open in the desensitized conformation(s). MTSET possesses a bulkier trimethylammonium headgroup and cannot cross membranes. We observed that MTSET significantly labels cysteine mutants at 6',2' and -1' when applied in the open state $(71 \%, 75 \%, 46 \%$ respectively). Applications in the desensitized state yielded a small variable inhibition at 2' $(12 \pm 10 \%)$ and none at 6 ', indicating that access to these positions is easier for MTSEA than for the bulkier MTSET. Put together, we favor the scheme where I1 is assigned to a pre-active state. Still desensitization is a complex process involving several distinct states ${ }^{15}$ and we cannot rule out the possibility that I1 represents a desensitized state.

The challenge of matching structures to states without ambiguity transcends the present study and pertains to the whole pLGIC structural field ${ }^{2,27,28}$. This challenge arises from 
diverse factors: limited resolution, putative influence of the detergent, of crystal packing, of receptor engineering - and the possibility that ensembles of multiple related conformations are necessary to properly depict a physiological state. Bearing in mind the ambiguities on state assignment, we compared the 5-HT3 transitions to those observed for $\mathrm{GLIC}^{4}, \mathrm{GluCl}^{29}$ and the glycine $a 1$ receptor (GlyR) ${ }^{7}$. Common agonist-induced features emerge, such as a global twist, an ECD quaternary reorganization, a interface rearrangement between domains involving the conserved sandwich motif, and local motions of the upper TMD. Differences also appear: the extent of TMD reorganisation seen in $\mathrm{F}$, with $\mathrm{M}^{4}$ sliding on other helices, is not observed in other receptors; the ECD reorganisation is well described as 'un-blooming, 4 for GLIC and GluCl, but not for the 5HT3 and glycine receptors. The F open pore is wider than that of GLIC and narrower than that of GlyR. Bacterial, animal anionic, animal cationic channels may, thus, have evolved distinct sets of conformations for a given physiological state, as they belong to separate branches of the pLGIC family. Our new 5-HT3 structures shed light on several transitions in the cationic branch. They also contribute to advance our knowledge on other important aspects of pLGIC research, which are only alluded to in this report, such as the role of M4 in gating, the pharmacology of allosteric sites, the lower TMD and ICD dynamics. Further work with better resolution, structures of mutants and in complex with other ligands, will certainly complement and ascertain mechanistic insights, which may, nevertheless, remain incomplete until kinetic structural experiments come of age.

\section{Methods}

\section{Protein expression}

The wild-type mouse 5-HT3A receptor was expressed in a stable, inducible cell line derived from HEK T-REx 293 cells (ThermoFisher), as previously described ${ }^{5,30,31}$. An inducible cell line was cultured in suspension in flasks in an orbital incubator (typical culture size: 5 liters). The protein expression was induced when cells reached $2.10^{6}$ cells $/ \mathrm{mL}$. Valproic acid was added one day later and cells were cultured for one more day. Cells were then pelleted by low-speed centrifugation, frozen and kept at $-80^{\circ} \mathrm{C}$.

\section{Protein purification}

In a typical purification batch, $20 \mathrm{~g}$ of cells were resuspended in buffer A (10 mM HEPES pH 7.4, 1 mM EDTA, antiprotease cocktail; $10 \mathrm{~mL}$ of buffer per gram of cells) mechanically lysed (Ultraturrax T20, 6x30 seconds) and membranes were collected by ultracentrifugation (100'000g for 1 hour). All steps were carried at $4^{\circ} \mathrm{C}$. Membranes were resuspended in buffer B (50 mM Tris pH 8, $500 \mathrm{mM} \mathrm{NaCl}$, antiproteases cocktail, $25 \mathrm{~mL}$ of buffer per gram of membrane) and the solution was supplemented with $0.15 \%$ of C12E9 for solubilisation using gentle stirring ( 1.5 hours). The insoluble material was removed by ultracentrifugation ( 100 ' $000 \mathrm{~g}$ for 45 minutes). Solubilized proteins were purified by affinity chromatography using gravity flow Streptactin resin (IBA, typically $25 \mathrm{~mL}$ of resin), eluted in buffer C (50 $\mathrm{mM}$ Tris $\mathrm{pH} 7.5,125 \mathrm{mM} \mathrm{NaCl}, 0.01 \%$ C12E9) and concentrated to $\sim 0.5 \mathrm{mg} / \mathrm{mL}$ using Millipore $100 \mathrm{kDa}$ cut-off devices. The purification tag was cleaved, and the carbohydrates were digested by addition of $0.04 \mathrm{mg}$ of TEV protease and $0.1 \mathrm{mg}$ of PNGase F per $1 \mathrm{mg}$ of 
protein with gentle stirring overnight. The protein was further concentrated and then applied to a Superose 6 column (GE healthcare) equilibrated in buffer $\mathrm{C}$.

\section{Electron microscopy}

The most homogeneous fraction of 5-HT3 receptor after the SEC were concentrated to $\sim 1.5$ $\mathrm{mg} / \mathrm{mL}$ (in the best cases, no concentration was required). The sample was mixed with lipids ( $0.01 \%$ phosphatidic acid, $0.01 \%$ cholesterol hemisuccinate, $0.01 \%$ brain phosphatidylcholine, Avanti Polar lipids) and ligands: $2 \mathrm{mM}$ tropisetron (Tocris); or $50 \mu \mathrm{M}$ serotonin and $2 \mathrm{mM}$ of calcium (conditions known to promote fast desensitization ${ }^{32,33}$ ); or $30 \mu \mathrm{M}$ serotonin and $100 \mathrm{mM}$ of TMPPAA (Sigma-Aldrich). Samples were incubated for 10-30 minutes on ice. $3.5 \mu \mathrm{l}$ were deposited on a glow-discharged $(30 \mathrm{~mA}, 50 \mathrm{sec})$ Quantifoil copper-rhodium 1.2/1.3 grid, blotted for 10 seconds at force 0 using a Mark IV Vitrobot and plunge-frozen in liquid ethane. 4-10 grids were screened during each data collection, as ice thickness varied between grids. Optimization was performed on a inhouse Polara electron microscope. Datasets were recorded on Titan Krios electron microscopes with K2 cameras at C-CINA (Basel, Switzerland) or at the ESRF (Grenoble, France). Details of data collections are found in Extended Data Fig. 2.

\section{Image processing}

At the Basel Krios microscope, the data collection was monitored online and good images were selected using Focus ${ }^{34}$; images were acquired in super-resolution mode and binned by Fourier-space cropping during the drift correction. At the ESRF Krios microscope the counted mode was used. Drift was corrected with MotionCor $2^{35}$ and dose-weighted sums were used for subsequent processing, except for CTF correction, which was performed using $\mathrm{GCTF}^{36}$ on non dose-weighted sums. Picking was performed with Gautomatch (http:// www.mrc-lmb.cam.ac.uk/kzhang/Gautomatch/) using average from 2D classes as templates. Subsequent steps were performed using Relion ${ }^{37}$ on a GPU workstation. Typically two rounds of 2D classifications, with 20-30 classes were performed, followed by a round of 3D classification without imposing symmetry (3-6 classes) with a low-pass filtered initial model of the receptor. Particles presenting five-fold symmetry were selected, submitted to 3D classifications (classifications and data processing are further described in Extended Data Fig. 3,5) and the best sets of particles were then subjected to 3D auto-refinement. In the post-processing step, a soft mask was calculated and applied to the two half-maps before the Fourier shell coefficient (FSC) was calculated. Map sharpening (B-factor fixed at -100 $\AA^{2}$ for $\mathrm{I} 1$ and $\mathrm{F}$, automatic estimation for $\mathrm{T}$ and $\mathrm{I} 2$ ) was also performed in the post-processing step. We tried the Phenix auto-sharpen programm ${ }^{38}$, which only improved the F map. The quality of the final reconstructions are illustrated in Extended Data Fig. 6.

\section{Model refinement and structure analysis}

Refinement was performed with the Phenix suite ${ }^{39}$. Cycles of real-space refinement were performed using global minimization, rigid body fit, local rotamer fitting (and B-factor refinement in late stages) in alternance with manual rebuilding in $\operatorname{Coot}^{40}$. NCS and secondary structure restraints were enabled. The 4 models comprise residues 10-307 and 426-460. T, I1 and I2 also have Mx and MA residues 308-330 and 399-426, which due to flexibility could not be build in F. Tropisetron (numbered 902) was placed in the orthosteric 
side of T, and serotonin (numbered 901) was placed in the site of I1, F and I2. The densities for serotonin in I1 and F permit to build the ligand in several equivalent orientations, and we used the unambiguous density in I 2 to choose the same ligand pose in these 3 structures. The stereochemical properties of the final models, analysed with the Molprobity server (http:// molprobity.biochem.duke.edu/), are reported in Extended Data Fig. 2. Pore profiles were plotted using HOLE ${ }^{41}$, RMSD were calculated with Superpose in the CCP4 suite ${ }^{42}$. Figures were prepared with PyMOL Molecular Graphics System (Schrodinger), Chimera ${ }^{43}$ or CueMol.

\section{Molecular dynamics}

Molecular-assays were built for the four conformational states of 5-HT3 described in the manuscript. For F, I1 and I2 we used the reported cryo-EM structures whereas for the inhibited conformation, we employed the crystal structure (PDB:4PIR) because of its higher resolution. The co-crystallized nanobodies were removed and the protein was modeled without any serotonin. The five serotonins present in F, I1 and I2 structures were kept in the models. For I2, since no obvious densities were observed for TMPPAA, it was not represented in the model. The intracellular domain of the F conformation (not resolved in the cryo-EM density) was not included in the model.

Using the CHARMM-GUI web-interface ${ }^{44,45}$, each structure was embedded in a fully hydrated palmitoyl-oleyl-phosphatidylcholine (POPC) bilayer consisting of ca. 240 lipid units and ca. 30,000 for F and 42,000 water molecules for T, I1 and I2. A concentration of $150 \mathrm{mM}$ of $\mathrm{K}^{+} \mathrm{Cl}^{-}$was explicitly added to each system, with ensuring their electric neutrality. The all-atom CHARMM36 force field ${ }^{46}$ and revision thereof for lipids ${ }^{47}$ were used to describe the system and CMAP corrections were introduced for the protein ${ }^{48}$. For water, we used the TIP3P model ${ }^{49}$. A subset of mass of the heavy atoms of lipids, protein and serotonin were transferred on the hydrogens atoms to which they are bound, to reach a hydrogen mass of $3.024 \mathrm{Da}$. Chad et al. ${ }^{50}$ demonstrated that, using such a mass repartitioning scheme, the equation of motions can be integrated with a time step up to $4 \mathrm{fs}$ without modifying the dynamics and the thermodynamics of the system.

All simulations were carried out with the NAMD package (version 2.12) ${ }^{51}$. Simulations were performed in the isothermal-isobaric ensemble at $\mathrm{T}=300 \mathrm{~K}$ and $\mathrm{P}=1$ atm with anisotropic scaling of the simulation cell ${ }^{52}$ and long-range electrostatic interactions were treated with the particle-mesh Ewald method ${ }^{53}$ and short-range electrostatics and LennardJones interactions were smoothly truncated. The equations of motion were integrated with a time step of 4 and 8 fs for short- and long-range forces, respectively, using the Verlet rRESPA multiple time-step propagator ${ }^{54}$. Covalent bonds involving hydrogen atoms were constrained to their equilibrium length by means of the Rattle/shake algorithm ${ }^{55,56}$ and the Settle algorithm for water ${ }^{57}$. For each system, a smooth equilibration, along which the positions of the heavy atoms of the protein were restrained harmonically, was carried out during $60 \mathrm{~ns}$. After releasing the restraints, the simulations were extended up to $1 \mu \mathrm{s}$. All analysis and molecular rendering were achieved with $\mathrm{VMD}^{58}$. Pore radii were inferred using the program HOLE ${ }^{41}$. 
The potentials of mean force (PMFs) underlying the translocation of a potassium ion in the I1 and F conformations of the homomeric 5-HT3A receptor were determined using a multiple-walker version ${ }^{59}$ of the adaptive biasing force (MW-ABF) algorithm ${ }^{60}$. For the I1 conformation, the reaction coordinate model was chosen as the Euclidian distance between the ion and the center of mass of the protein, projected onto its longitudinal axis, i.e., the $z$ direction of Cartesian space. In the case of the F conformation, a two-dimensional freeenergy landscape was generated, exploring ion diffusion in the pore not only longitudinally by means of the aforementioned projected Euclidian distance, but also radially. The PMF along the pore was recovered by integration of the marginal law in the radial direction. The reaction pathway was broken down in 14 and 12 windows for the I1 and F conformations, respectively. The free-energy landscapes were explored by four walkers, syncing gradients every $500 \mathrm{MD}$ steps. The total simulation time for the I1 and F conformations amounted to 2.16 and $3.04 \mu \mathrm{s}$, respectively, wherein the last $0.8 \mu$ s were utilized to estimate the error bars associated to the PMFs, based on an independent mapping of the free-energy landscape by the walkers.

\section{Electrophysiology}

Electrical recordings were obtained by two-electrode voltage-clamp (TEVC) on Xenopus oocytes expressing either WT or mutated homomeric m5-HT3A receptors. Mutants were obtained using the QuickChange Lightning (Stratagene) site-directed mutagenesis kit and oligonucleotides (Table S6) to introduce point mutations into the pcDNA5/TO-m5-HT3A plasmid. All the mutations were verified by sequencing.

Oocytes were prepared as previously reported ${ }^{61}$ using procedures that conformed to European regulations for animal handling and experiments, and were approved by governmental services (Authorization $\mathrm{N}^{\circ}$ D 3818510001 for the animal facility delivered by the Prefect of Isère) and the Institutional Ethics Committee (Ethics approval $N^{\circ} 12-040$ granted to Christophe Moreau by the Ethic Committee of Commissariat à l'Energie Atomique et aux Energies Alternatives). Difolliculated oocytes were injected by $30 \mathrm{~nL}$ of plasmid DNA (1 to $10 \mathrm{ng} / \mu \mathrm{L}$ ) coding for the desired 5-HT3A subunit (subcloned in pcDNA5 vector). Microinjected oocytes were incubated for 1 to 5 days at $19^{\circ} \mathrm{C}$ in Barth's solution (in mM: $1 \mathrm{KCl}, 0.82 \mathrm{MgSO}_{4}, 88 \mathrm{NaCl}, 2.4 \mathrm{NaHCO} 3,0.41 \mathrm{CaCl}_{2}, 16$ Hepes, pH 7.4) supplemented with $100 \mathrm{U} \cdot \mathrm{ml}^{-1}$ of penicillin and streptomycin.

Whole-cell TEVC recordings were obtained using an OC-725C Oocyte Clamp amplifier (Warner Instruments, Hamden, CT) at a constant $-50 \mathrm{mV}$ holding potential. Macroscopic currents were filtered at $1 \mathrm{kHz}$, digitized at $2 \mathrm{kHz}$ with a Digidata 1440 analog-to-digital interface and analyzed with Clampfit (Molecular Devices, Sunnyvale, CA). During the recordings, oocytes were constantly perfused with a 0 Ca-ND96 solution (containing in mM: $91 \mathrm{NaCl}, 2 \mathrm{KCl}, 1 \mathrm{MgCl}_{2}, 5 \mathrm{HEPES}, 1 \mathrm{EGTA}, \mathrm{pH} 7.4$ ) at a rate of $1.3 \mathrm{~mL} / \mathrm{min}$, which allowed application of serotonin and other compounds in the sec time range (20 fold concentration change of applied compounds in $5 \mathrm{sec}$ ). Throughout this study, electrophysiological responses were induced by $10 \mu \mathrm{M}$ serotonin (a saturating dose for all tested constructions, not shown) and peaked in less than $3 \mathrm{sec}$. In all constructions tested, 10 min applications of serotonin induced almost complete desensitization (to less than $3 \%$ of 
the peak current with $t_{1 / 2}$ in the min range). Full recovery of desensitization was observed after washing serotonin for $10 \mathrm{~min} . \mathrm{Ca}^{2+}$ ions are permeant through 5-HT3 receptors ${ }^{62}$. Calcium was thus carefully removed from the recording perfusion solution to avoid contamination of the serotonin-induced responses by endogenous oocyte current secondarily activated by $\mathrm{Ca}^{2+}$ ions entering the oocyte through the serotonin receptors.

MTSEA (2-aminoethyl methanethiosulfonate bromide) and MTSET (2trimethylammonium-ethyl methanethiosulfonate bromide) were purchased from Interchim (Montluçon, France) and prepared immediately before perfusion from stock solutions in water stored at $-20^{\circ} \mathrm{C}$. The effect of MTS compounds on pore cysteine-mutants was studied using the following protocol: (i) checking stability of the response with a train of three to five applications of $10 \mu \mathrm{M}$ serotonin for $10 \mathrm{sec}$, every $5 \mathrm{~min}$ ( $\mathrm{I}_{\text {pre }}$ ); (ii) applying MTS compounds for $2 \mathrm{~min}$ at $1 \mathrm{mM}$, either in the absence of 5-HT (to probe cysteine accessibility in the resting state), simultaneously with $10 \mu \mathrm{M} 5-\mathrm{HT}$ (to probe the open state) or simultaneously with $10 \mu \mathrm{M}$ 5-HT after a $10 \mathrm{~min}$ pre-application of 5-HT alone allowing for complete desensitization; (iii) following the effect of MTS compounds during a second train of five applications of 5-HT for $10 \mathrm{sec}$ every $5 \mathrm{~min}\left(\mathrm{I}_{\text {post }}\right)$. Irreversible effects of MTS compounds were quantified by measuring $\mathrm{I}_{\text {post }} 25 \mathrm{~min}$ after removal of the MTS compound. The percentage of inhibition or potentiation was calculated as $\left\langle\left(\mathrm{I}_{\mathrm{pre}}-\mathrm{I}_{\mathrm{post}}\right) / \mathrm{I}_{\mathrm{pre}} * 100\right\rangle$. MTS compounds (applied at $1 \mathrm{mM}$ simultaneously with $10 \mu \mathrm{M}$ serotonin) have no detectable effect on WT receptors (not shown).

\section{Voltage Clamp Fluorometry}

VCF recordings were made on Xenopus oocytes provided by Ecocyte Bioscience (Germany), injected with $50 \mathrm{ng} / \mu \mathrm{L}$ of plasmid DNA encoding for the m5-HT3A receptor mutant $\mathrm{S} 19^{\prime} \mathrm{C}$ and after 2 to 6 days of expression in Barth solution at $17^{\circ} \mathrm{C}$. They were labeled with MTS-TAMRA (Toronto chemicals) $5 \mathrm{~min}$ at $17^{\circ} \mathrm{C}$ in ND96 buffer without $\mathrm{CaCl} 2+10 \mu \mathrm{M} 5-\mathrm{HT}$, then rinsed and stored at $17^{\circ} \mathrm{C}$ up to $4 \mathrm{~h}$ before recording in ND96. Recording were made in a TEVC set up previously described ${ }^{13}$ and adapted for fluorescence recording. Briefly recordings were done with a VCF dedicated chamber with only a fraction of the oocyte perfused and illuminated by a LED (coolled PE-4000). Light was collected using a fluorescence microscope (Zeiss Axiovert135) equipped with a 40x objective (Plan neofluar), a TRITC filter set and a photomultiplier tube (Hamamatsu photonics). Recordings were made at $-60 \mathrm{mV}$ clamp, $500 \mathrm{~Hz}$ sampling rate with a $550 \mathrm{~nm}$ excitation wavelength. Data were filtered, corrected for baseline and photobleaching when necessary and analyzed using pClamp and Axograph. Dose response curves were fitted to the Hill equation I/Imax = $1 /\left(1+\left(\mathrm{EC}_{50} /[5-\mathrm{HT}]\right)^{\mathrm{nH}}\right)$ using Prism. Serotonin dose-response relations were shifted to the left with half-responses for fluorescence and current at 40 and $240 \mathrm{nM}$ respectively (the $\mathrm{EC}_{50}$ of the wild-type receptor is $800 \mathrm{nM}$ ).

\section{FLIPR Membrane Potential Blue (FMP) assay}

The agonist properties of 5-HT and TMPPAA (Sigma-Aldrich) were characterized at human wild-type or mutant 5-HT3A receptors transiently expressed in tsA201 cells in the FMP assay. The generation of some of the h5-HT3A mutants have been described previously 63 . Other mutants were constructed by introduction of point mutations into the h5-HT3A- 
pCIneo plasmid using Quikchange II XL site-directed mutagenesis (Stratagene) and oligonucleotides (TAG Copenhagen), and the absence of unwanted mutations in all cDNAs created by PCR was verified by sequencing (Eurofins MWG Operon). The cells were cultured in Dulbecco's Modified Eagle Medium supplemented with penicillin (100 U/ml), streptomycin $(100 \mathrm{mg} / \mathrm{ml})$ and $10 \%$ fetal bovine serum in a humidified atmosphere of 5\% $\mathrm{CO}_{2}$ and $95 \%$ air at $37^{\circ} \mathrm{C} .1 .2 \times 10^{6}$ cells were split into a $6 \mathrm{~cm}$ tissue culture plate, transfected the following day with a total of $4 \mu \mathrm{g}$ cDNA (WT or mutant h5-HT3A-pCIneo) using Polyfect (Qiagen) as transfection reagent, and split into poly-D-lysine-coated black 96-wells plates ( $8 \times 10^{4}$ cells/well) with clear bottom (BD Biosciences) the following day. 20-24 $\mathrm{h}$ after the transfection, the medium was aspirated, and the cells were washed with $100 \mu \mathrm{l}$ Krebs buffer $\left(140 \mathrm{mM} \mathrm{NaCl}, 4.7 \mathrm{mM} \mathrm{KCl}, 2.5 \mathrm{mM} \mathrm{CaCl}_{2}, 1.2 \mathrm{mM} \mathrm{MgCl}_{2}, 11 \mathrm{mM}\right.$ HEPES, $10 \mathrm{mM}$ D-Glucose, pH 7.4). Then $100 \mu \mathrm{K}$ Krebs buffer supplemented with 0.5 $\mathrm{mg} / \mathrm{ml} \mathrm{FMP} \mathrm{assay} \mathrm{dye} \mathrm{(Molecular} \mathrm{Devices)} \mathrm{was} \mathrm{added} \mathrm{to} \mathrm{each} \mathrm{well,} \mathrm{and} \mathrm{the} \mathrm{96-well} \mathrm{plate}$ was assayed at $37^{\circ} \mathrm{C}$ in a FLEXStation3 Benchtop Multi-Mode Microplate Reader (Molecular Devices) measuring emission [in fluorescence units (FU)] at $565 \mathrm{~nm}$ caused by excitation at $530 \mathrm{~nm}$ before and up to $90 \mathrm{sec}$ after addition of $33.3 \mu \mathrm{l}$ assay buffer supplemented with agonist (5-HT or TMPPAA). The experiments were performed in duplicate at least three times for each agonist at all receptors. Concentration-response curves for the agonists were constructed based on the difference in the fluorescence units (rFU) between the maximal fluorescence recording made before and after addition of the agonists at different concentrations. The curves were generated by non-weighted least-squares fits using the program KaleidaGraph (Synergy Software).

\section{Extended Data}




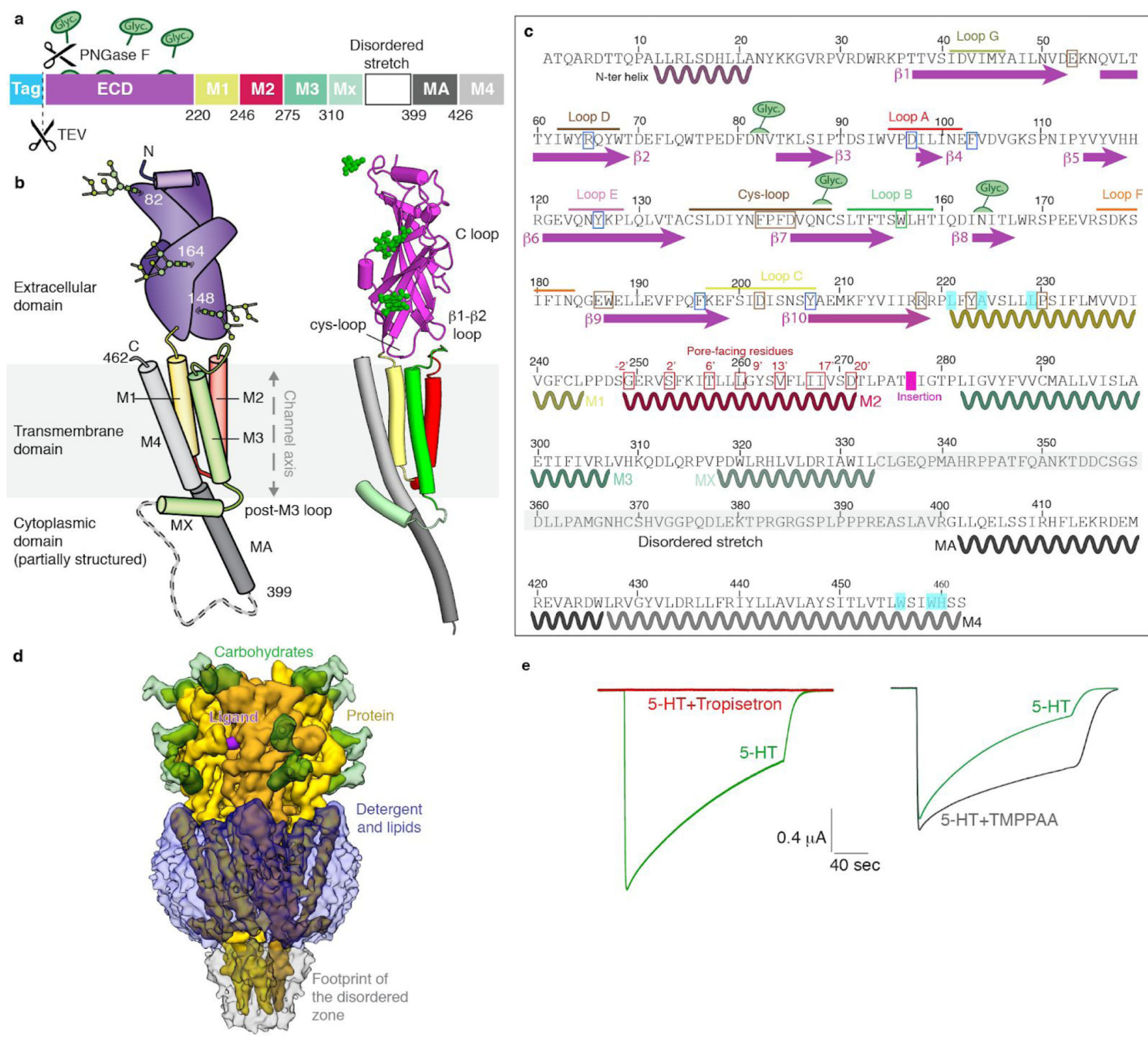

Extended Data Figure 1. 5-HT3 receptor topology and sequence

a. The full-length receptor elements are schematized. The color code is the same for all panels. Scissors indicate the enzymatic treatments used during purification: purification tag removal using TEV protease, and partial removal of carbohydrates using PNGase F.

b. Topology scheme and structure of one subunit of the 5-HT3 receptor.

c. Sequence and numbering of the mouse 5-HT3A receptor used in the present study.

Secondary structures and important loops are indicated. Neurotransmitter site binding loops are highlighted. Important residues discussed in the study are boxed (pore-facing in red, sandwich motif in brown, neurotransmitter site in blue). Cyan highlights residues important for TMPPAA potency. Glycosylation sites are depicted in green. The mouse receptor used for this study is a variant compared to the consensus sequence as it contains an alanine 
insertion in the M2-M3 loop, which is highlighted by the pink box. Notably, this insertion is present in the consensus human receptor. This panel is adapted from ${ }^{5}$.

d. A typical unmasked reconstruction, with different density levels overlaid depicting the protein itself (yellow), ligands (purple), linked glycans (green), the detergent-lipid belt (transparent purple) and the footprint of the disordered zone (transparent grey).

e. Currents induced in Xenopus oocytes expressing the mouse 5-HT3 receptor by a 3-minute application of $10 \mu \mathrm{M}$ serotonin (green traces), co-application of $10 \mu \mathrm{M}$ serotonin and 100 $\mathrm{nM}$ tropisetron (following a 5-minute pre-application of tropisetron, red trace), coapplication of $10 \mu \mathrm{M}$ serotonin and $10 \mu \mathrm{M}$ of TMPPAA (following a 5-minute preapplication of TMPPAA, black trace). Current traces are representative of 3 independently repeated experiments. 


\begin{tabular}{|c|c|c|c|c|}
\hline & Tropisetron & \multicolumn{2}{|c|}{ Serotonin } & $\begin{array}{l}\text { Serotonin and } \\
\text { TMPPAA }\end{array}$ \\
\hline \multicolumn{5}{|l|}{ Dat a collection and processing } \\
\hline Microscope & Krios@CCINA & \multicolumn{2}{|c|}{ Krios@CCINA } & Krios@ESRF \\
\hline Magnification & 130,000 & \multicolumn{2}{|c|}{130,000} & 130,000 \\
\hline Voltage (kV) & 300 & \multicolumn{2}{|c|}{300} & 300 \\
\hline Eectron exposure $\left(\mathrm{e}-/ \AA^{2}\right\}$ & $\sim 80$ & \multicolumn{2}{|c|}{$\sim 60$} & $\sim 50$ \\
\hline Defocus range $(\mu \mathrm{m})$ & $0.5-2.5$ & \multicolumn{2}{|c|}{$0.8-2.5$} & $0.8-2.5$ \\
\hline Pixel size $(\AA)$ & 0.52 & \multicolumn{2}{|c|}{0.52} & 1.067 \\
\hline Symmetry imposed & C5 & \multicolumn{2}{|c|}{$\mathrm{C} 5$} & $\mathrm{C} 5$ \\
\hline Number of images & $\sim 1500$ & \multicolumn{2}{|c|}{$\sim 2000$} & $\sim 3000$ \\
\hline Particles picked & $160 \mathrm{k}$ & \multicolumn{2}{|c|}{$456 \mathrm{k}$} & $250 \mathrm{k}$ \\
\hline Symmetric-looking particles & $133 \mathrm{k}$ & \multicolumn{2}{|c|}{$145 \mathrm{k}$} & $62 \mathrm{k}$ \\
\hline Particles refined & 43,558 & 16,660 & 10,667 & 62,032 \\
\hline Refinement & T & 11 & $\mathrm{~F}$ & 12 \\
\hline Initial model used & $4 \mathrm{PIR}$ & $4 \mathrm{PIR}$ & 4PIR & $4 \mathrm{PIR}$ \\
\hline Resolution $(\AA)$ & 4.5 & 4.2 & 4.1 & 3.2 \\
\hline FSCthreshold & 0.143 & 0.143 & 0.143 & 0.143 \\
\hline Map sharpening B-factor $(\AA \hat{\}})$ & -247 & -100 & -100 & -127 \\
\hline \multicolumn{5}{|l|}{ Model composition } \\
\hline Non-hydrogen protein atoms & 15,645 & 15,670 & 13,805 & 15,670 \\
\hline Protein residues & 1,935 & 1,925 & 1,675 & 1,925 \\
\hline Ligands & 585 & 415 & 415 & 415 \\
\hline \multicolumn{5}{|l|}{ B-factor (Å } \\
\hline protein & 127 & 130 & 83 & 78 \\
\hline ligand & 113 & 102 & 73 & 53 \\
\hline \multicolumn{5}{|l|}{ RM.Sdevitions } \\
\hline Bond lengths $(\AA)$ & 0.008 & 0.009 & 0.009 & 0.006 \\
\hline Bond angles $\left(^{\circ}\right)$ & 1.5 & 1.1 & 1.2 & 1.0 \\
\hline \multicolumn{5}{|l|}{ Validation } \\
\hline Molprobity score & 2.0 & 1.45 & 1.35 & 1.18 \\
\hline Gashscore & 14.1 & 8.3 & 6.4 & 3.9 \\
\hline Poor rotamers (\%) & 0.3 & 0.2 & 0.3 & 0.3 \\
\hline \multicolumn{5}{|l|}{ Ramachandran plot } \\
\hline Favored (\%) & 95.1 & 98.0 & 98.5 & 98.4 \\
\hline Allowed (\%) & 4.9 & 2.0 & 1.5 & 1.6 \\
\hline Disallowed (\%) & 0.0 & 0.0 & 0.0 & 0.0 \\
\hline
\end{tabular}

Extended Data Figure 2. Statistics of 3D reconstructions and model refinement

Nature. Author manuscript; available in PMC 2019 July 08. 
a
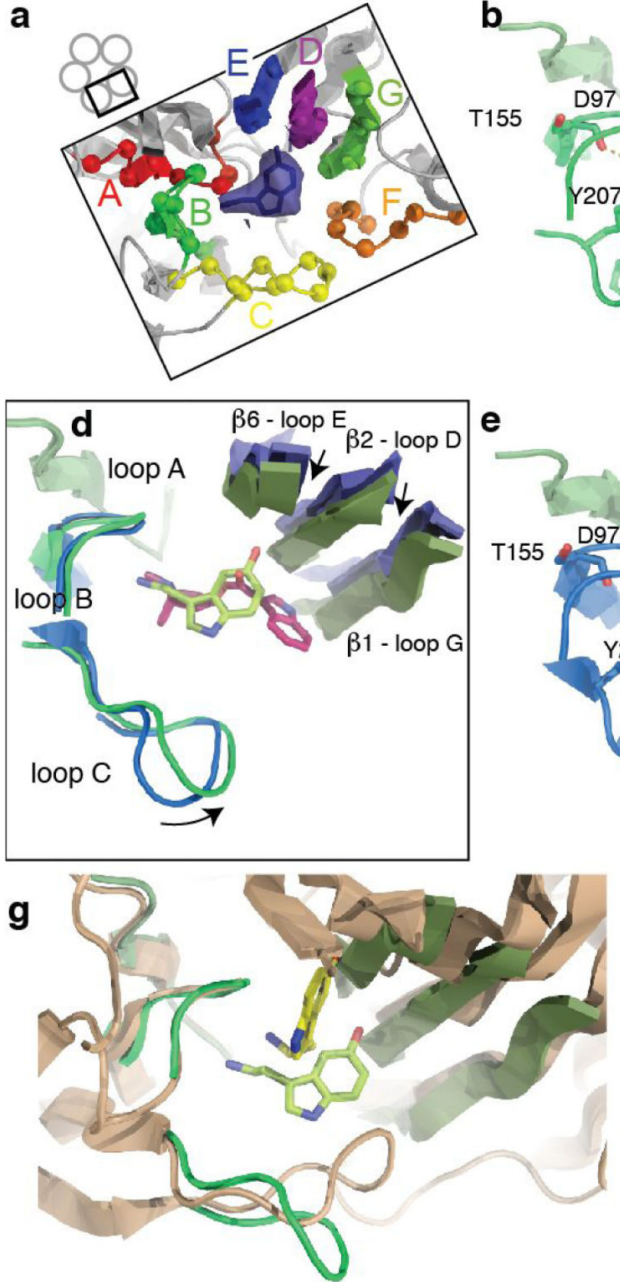

h

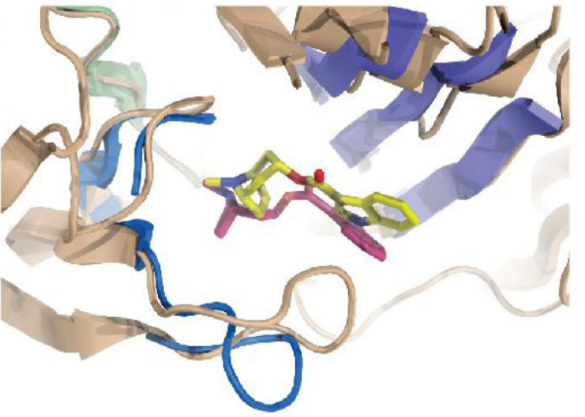

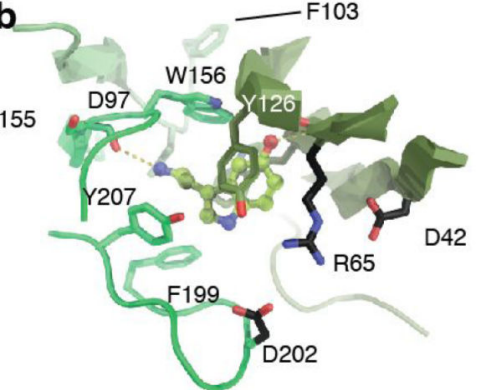

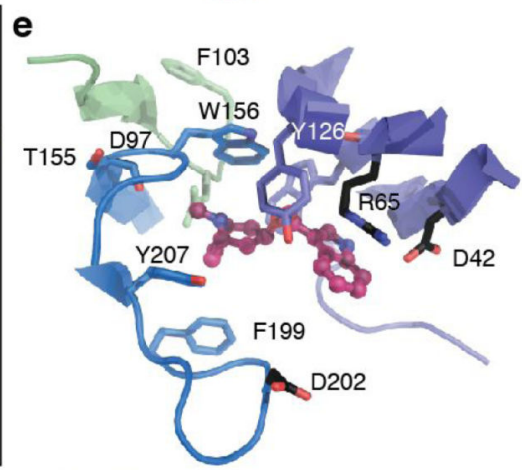

\section{f}

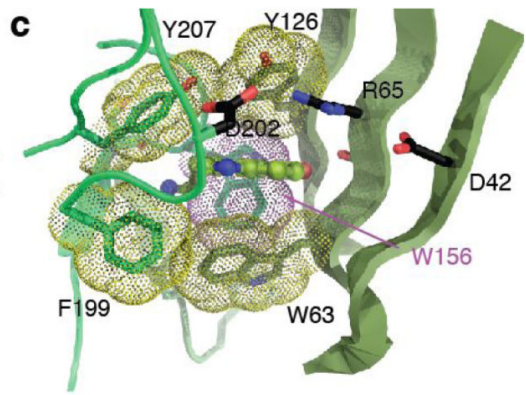

$f$
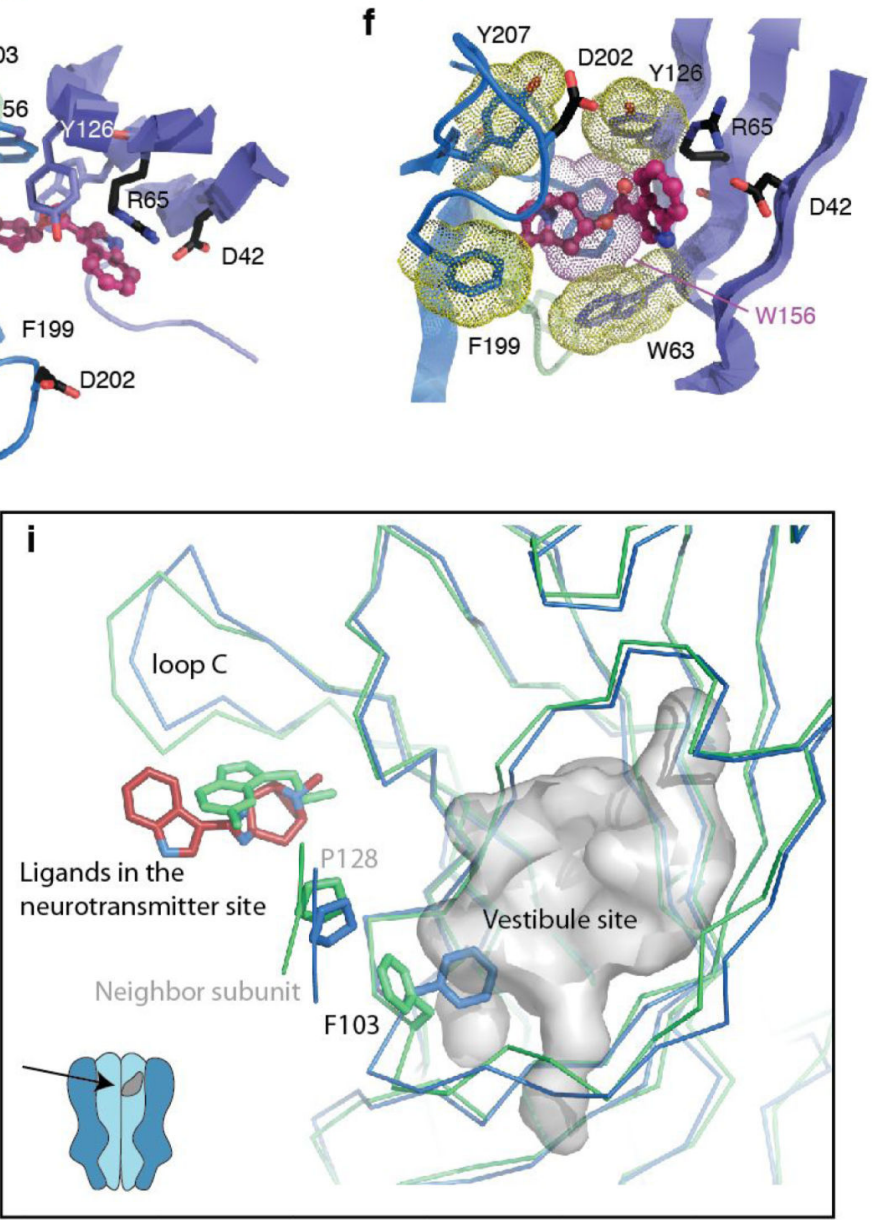

Extended Data Figure 3. Electron microscopy and 3D reconstructions for the tropisetron dataset (T), for the serotonin+TMPPAA dataset (I2)

a. Tropisetron dataset (T). Selection of 2D class averages from the set of particles used for refinement of the $\mathrm{T}$ reconstruction.

b. 3D reconstruction from the $\mathrm{T}$ dataset, filtered and colored according to RELION local resolution. A global and a cut-through side view are shown.

c. FSC curves for the density map before and after RELION post-processing, and between the model and the final map. 
d. Serotonin+TMPPAA dataset (I2). Selection of good 2D classes after one round of classification yields a set of $126 \mathrm{k}$ particules. One round of 3D classification with no symmetry imposed yield one class with pentameric symmetry, amounting to $62 \mathrm{k}$ particles. e. Unmasked unsharpened refined 3D reconstruction, filtered and colored according to RELION local resolution. The color range for resolution is similar to the equivalent representations for the other datasets in panel e and Extended Data Fig. 5c, allowing direct comparison.

f. FSC curves for the density map before and after RELION post-processing, and between the model and the final map.

g. On the left, a non-sharpened map filtered at $3.9 \AA$ resolution obtained with a subset of $55 \mathrm{k}$ particles coming from the $\mathrm{I} 2$ dataset, selected after further $3 \mathrm{D}$ classification focused on the ICD. The hypothetical trajectory of the polypeptide chain after Mx is bordered by a green line. No full connectivity can be visualized and the model was not built. It seems that the chain may abruptly go down after Mx, may interact with MA of the neighbouring subunit at the level of $\mathrm{H} 411$ (there is a clear blob there). Then it needs to join the beginning of MA but maps show no information how. The schematic representation highlights that this putative trajectory contacts the neighbour subunit MA close to residue H411. On the right, the corresponding sharpened and masked reconstruction, showing that model building is not possible. The density of the disordered stretch closed to H411 is highlighted by the pink ellipses. 
a
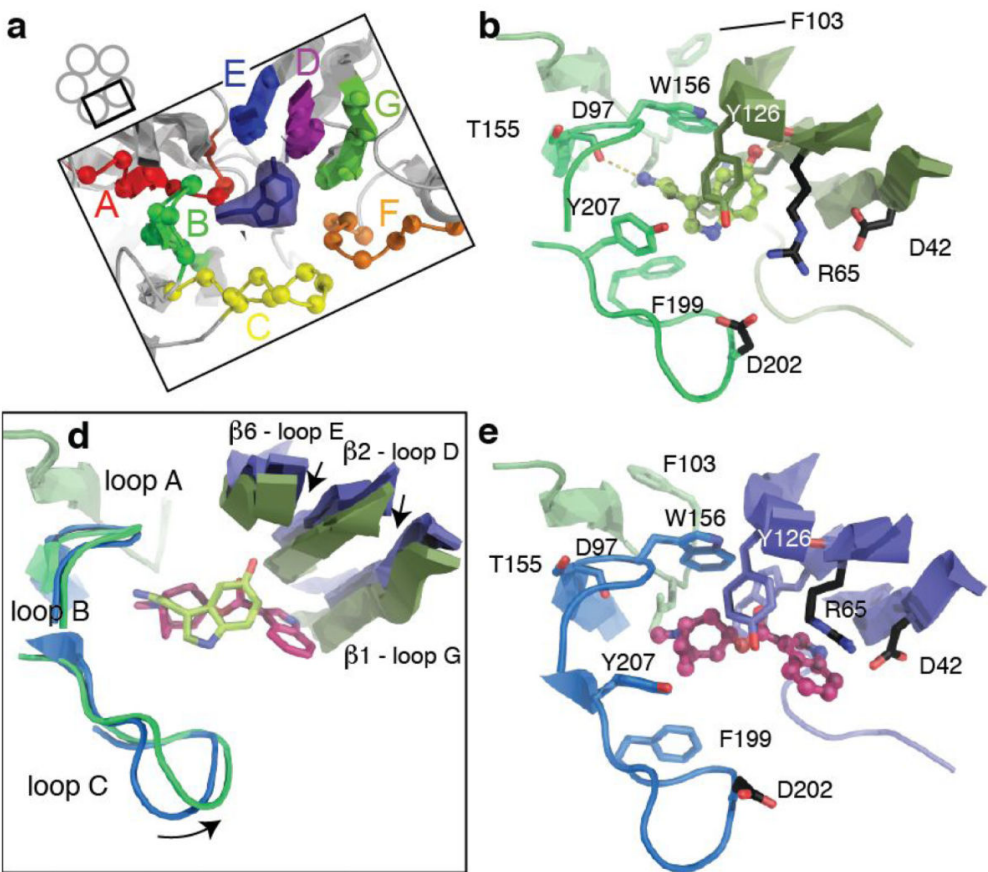

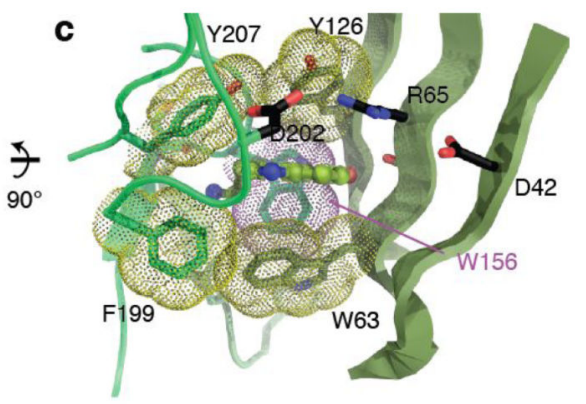

f

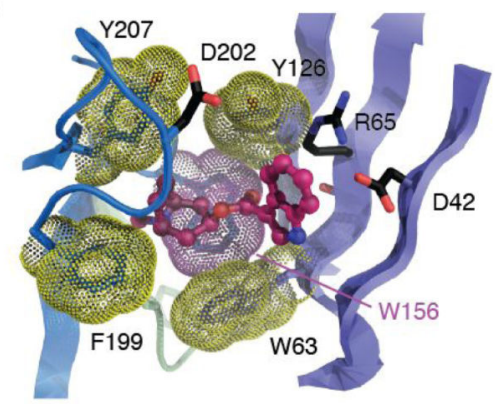

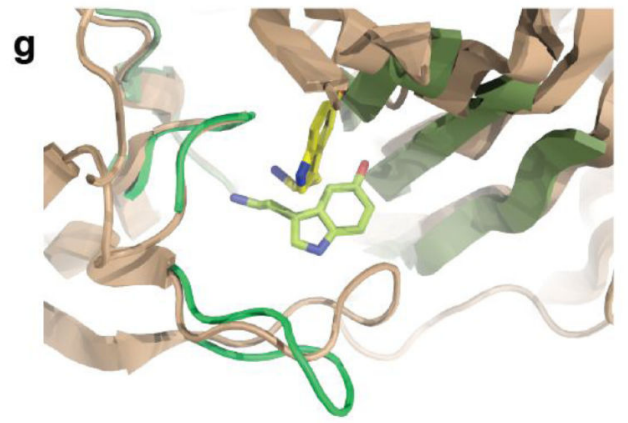

h
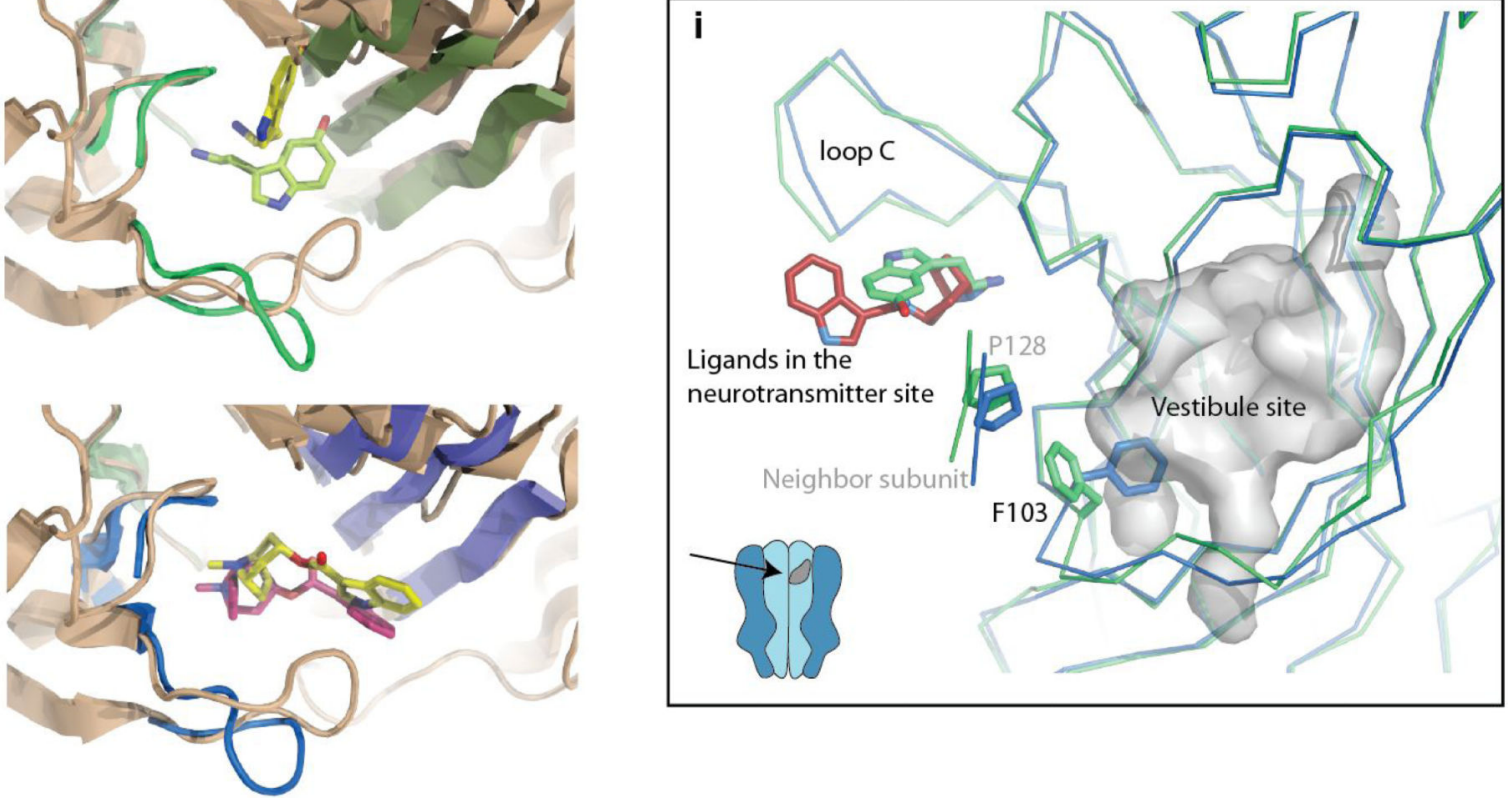

Extended Data Figure 4. Serotonin and tropisetron bound in the orthosteric site; vestibule allosteric site

a. Organisation of one of the 5 equivalent binding sites at the subunit interfaces, in the ECD.

Binding loops A-C are located on the principal subunit, binding elements D-G on the complementary subunit. The density around serotonin, in the I2 reconstruction, is shown as a transparent blue surface.

b and c. Serotonin (ball-and-stick) in the orthosteric site in the I2 structure. Aromatic residues (dotted surfaces in c.), hydrogen bonds with main chain atoms (dashed lines) and 
charged residues within salt-bridge distances (D202 on the principal side, R65 on the complementary side) are noted in two orthogonal views. Of note, mutants D202 $\mathrm{A}^{64}$ and $\mathrm{R} 65 \mathrm{~A}^{65}$ present impaired serotonin binding with 140 -fold and a $~ 50$-fold increase in Ki during competition assay for $\left[{ }^{3} \mathrm{H}\right]$-granisetron binding.

d. Superimposition of tropisetron-bound T (blue) and serotonin-bound I2 (green) structures, highlighting loop $\mathrm{C}$ motion and quaternary reorganization with arrows (principal subunit superimposition in this panel and in g. and h., observe complementary subunit $\beta$-strand shift). Serotonin (green) and tropisetron (magenta) are represented as sticks.

e and f. Tropisetron bound to the orthosteric site in the $\mathrm{T}$ structure. The bicyclic tropane moiety is sandwiched between W156 of loop B and Y207 of loop C, while the indole lies close to R65, W63 in loop D, D42 and I44 in loop G, and R169 in loop F. Functional exploration of the binding mode of tropisetron consistently showed that, among others, single mutations to cysteine of the aromatic residues W156, Y207, W63 abolished binding ${ }^{66}$. In order to fit tropisetron, the side chain of R65 is pushed upward and in turn displaces the side chains of Y67 and W168, compared to the empty orthosteric site of the Xray structure. Densities for these side chains are not well resolved.

g. Superimposition of the I2 structure with the crystal structure of a mutant AChBP (wheat cartoon) in complex with serotonin (yellow sticks) ${ }^{67}$ shows distinct binding modes.

h. Superimposition of the T structure with the crystal structure of AChBP (wheat cartoon) in complex with tropisetron (yellow sticks) shows a similar orientation of ligands but different interactions with the protein at loop C, D, E, F and G (not shown for clarity), in line with the 3 orders of magnitude difference in $\mathrm{Kd}\left(0.7\right.$ versus $\left.479 \mathrm{nM}^{68}\right)$.

i. Overlay of the T and I2 structures (in blue and green respectively) superimposed on a subunit ECD, showing the motion of F103 out of the vestibule site. The protein depicted as ribbon is viewed from inside the vestibule, and the intra-subunit cavity (in the I2 conformation) is represented as a grey surface. Acetate in GLIC, Flurazepam in the bacterial homologue $\mathrm{ELIC}^{69}$ or a drug fragment in a $a 7-\mathrm{AChBP}^{70}$ bind to this cavity. A putative rationale for the strong effect of $\mathrm{F} 103$ mutants on serotonin $\mathrm{EC}_{50}{ }^{71}$, despite its absence of participation to the binding site, comes from the observation of its concerted motion with that of strands from the neighbouring subunit the motion (around P128 and also around P110, the latter not represented here for clarity). Thus F103 motion may participate in subunit-subunit quaternary reorganisation and its motions exemplify the allosteric coupling between sites. 

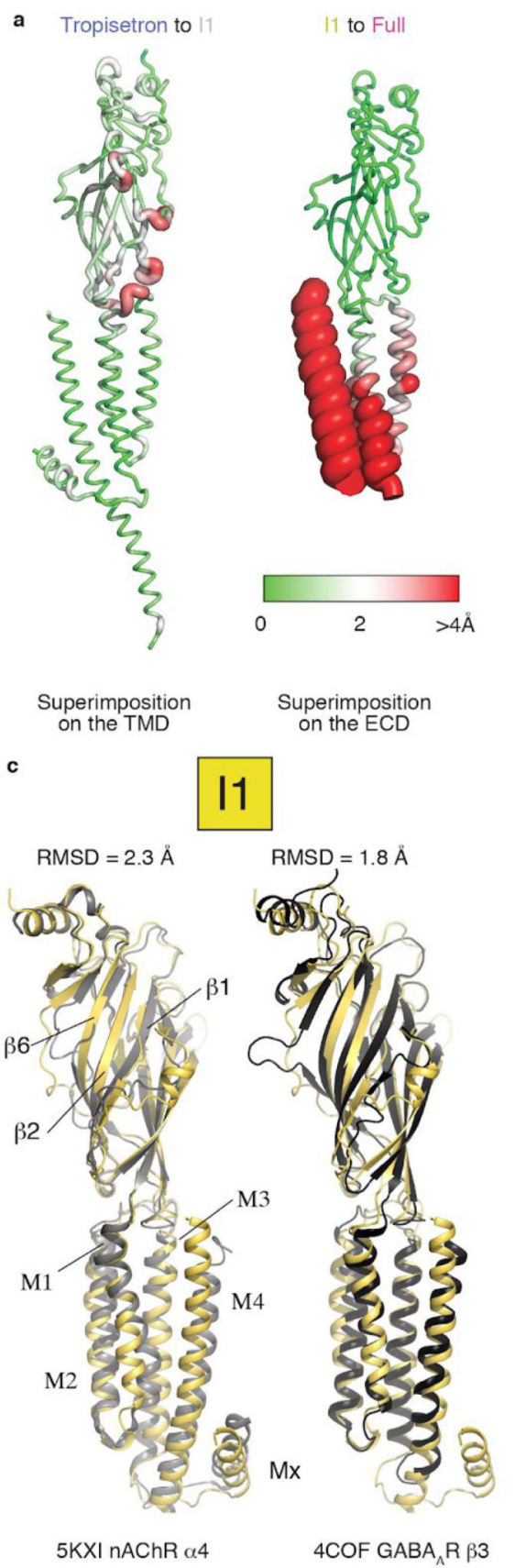

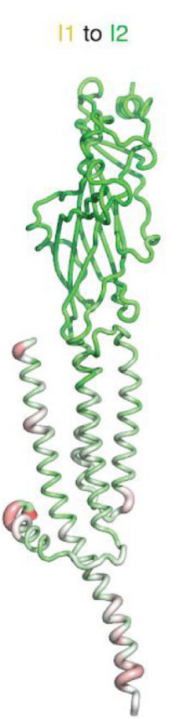

Superimposition on the ECD

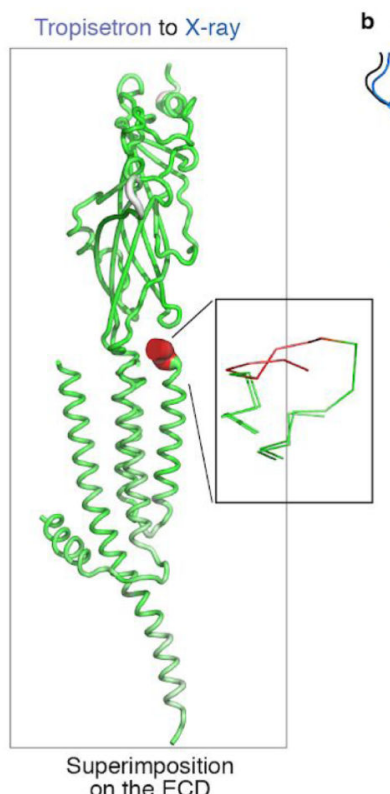

on the ECD
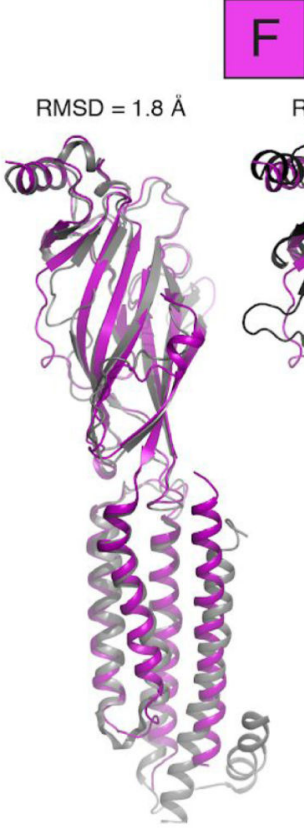

$5 \mathrm{KXI}$ nAChR $\alpha 4$

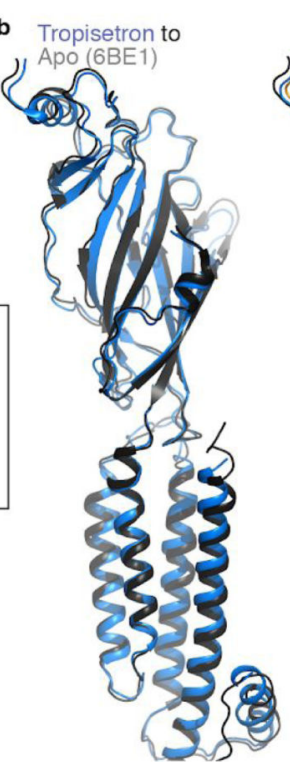

$\mathrm{RMSD}=1.15 \AA$

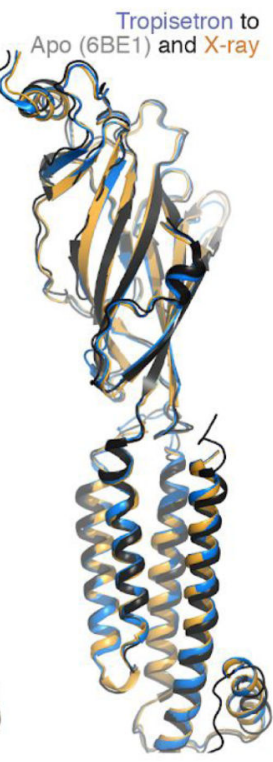

12

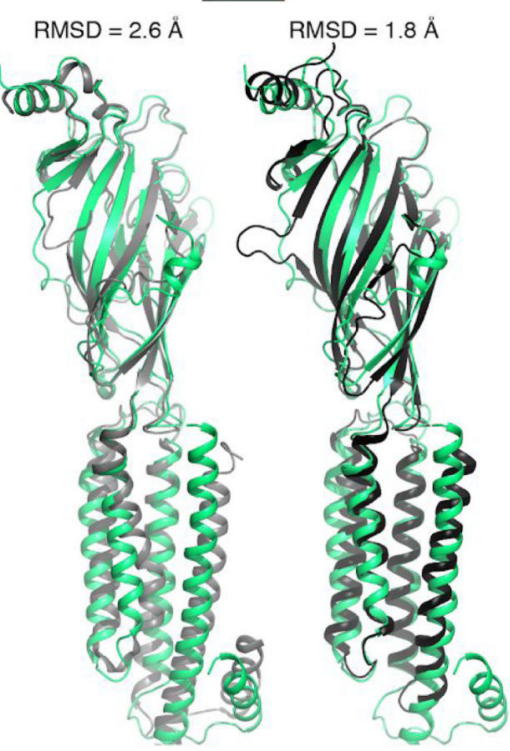

$5 \mathrm{KXI}$ nAChR $\alpha 4$

Extended Data Figure 5. Electron microscopy, classifications and 3D reconstructions for the serotonin dataset

a. Schematic representation of the data processing, with classical rounds of $2 \mathrm{D}$ and $3 \mathrm{D}$ classification yielding a consensus reconstruction seen parallel to the membrane plane (grey) where helices look like tubes of elliptic sections, indicating heterogeneity. Extensive classification trials, without alignment, with masks of various size covering the lower part of the TMD (shown in green and red), allow to distinguish two conformations (yellow, corresponding to the I1 structure and purple, corresponding to the F structure) representing the extreme positions of the helices in the consensus reconstruction. An overlay of the two 
reconstructions depicts the good superimposition of the ECD and the clear difference in the lower TMD. Grey rectangles indicate the positions of the slabs represented in panel $b$. All reconstructions shown are unmasked outputs of RELION 3D refinements.

b. Slabs, viewed perpendicular to the membrane plane, from the intracellular side showing the overlay of the I1 and the F reconstructions.

c. Reconstructions filtered and colored according to RELION local resolution. A global and a cut-through side view are shown.

d. Selection of 2D class averages from the set of particles used for refinement of the I1 and F reconstructions.

e. FSC curves showing the unmasked, masked FSCs (before and after post-processing in RELION) and the model-to-map FSC.

f. Selection of 'slice' views of the TMD of the final unmasked unsharpened reconstructions. 

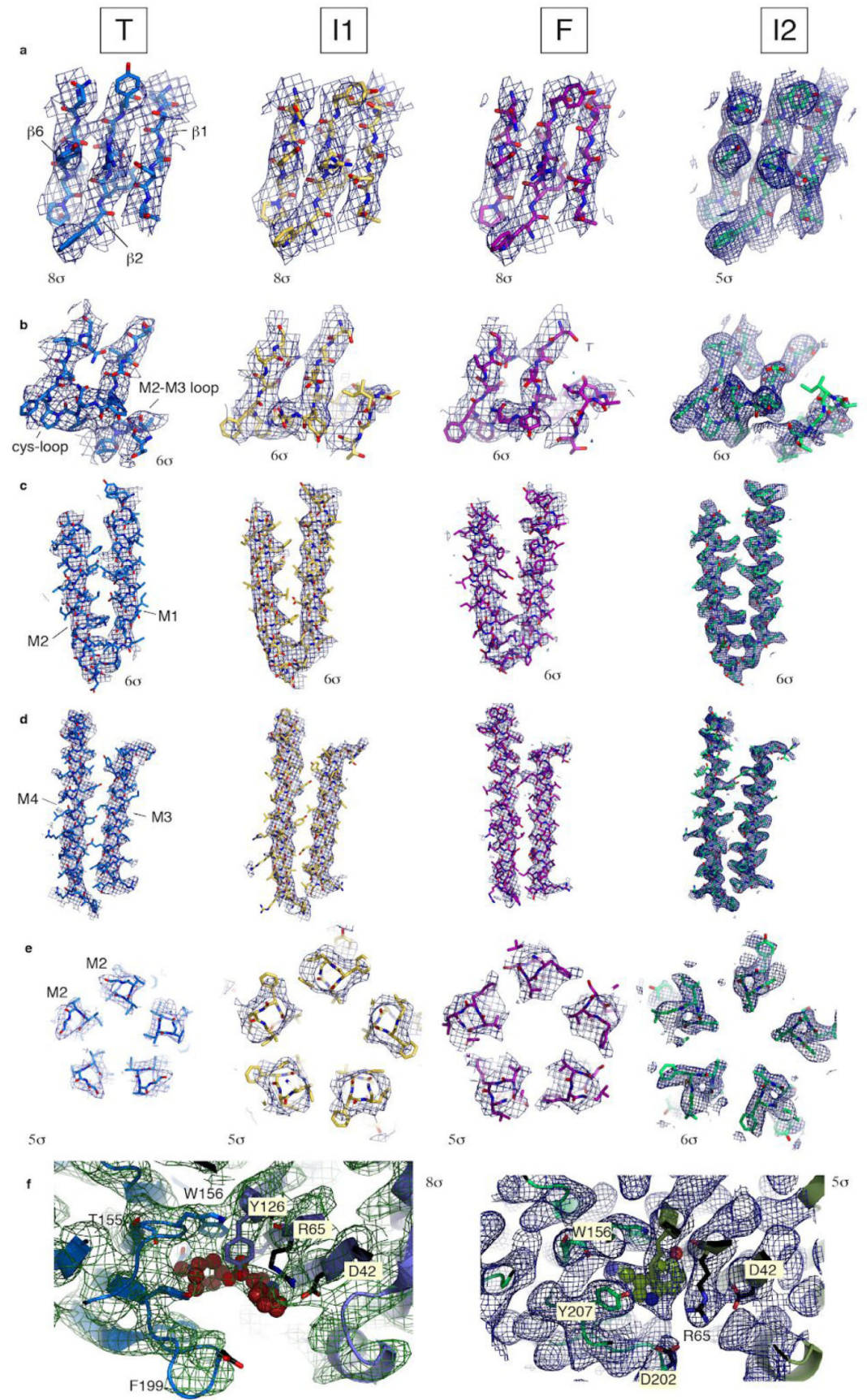

Extended Data Figure 6. Quality of the density maps, representative regions of the four 5-HT3 receptor reconstructions

Densities in mesh representations overlaid with structures for the T (blue), I1 (yellow), F (purple) and I2 (green) reconstructions and structures, from left to right in each panel. Views a-e are approximately the same as those in the equivalent figure of the $a 1 \mathrm{GlyR}$ article ${ }^{7}$ (Extended Data Fig. 4).

a. Representative densities of the $\beta$-sheets in the ECD.

b. Densities of Cys loop and the M2-M3 loop. 
c. Densities of helices M1 and M2.

d. Densities of M3 and M4.

e. Densities of M2 at the level of L9' (L260).

f. Densities around the ligands tropisetron (in T) and serotonin (in I2). The $3.2 \AA$ resolution of I2 permits unambiguous orientation of serotonin, and side chains of residues around the ligands are seen in densities. For tropisetron, given the limited $4.5 \AA$ global resolution, the orientation of the ligand and the position of surrounding side chain is less certain. The views are the same as in Extended Data Fig. 43b,e. 

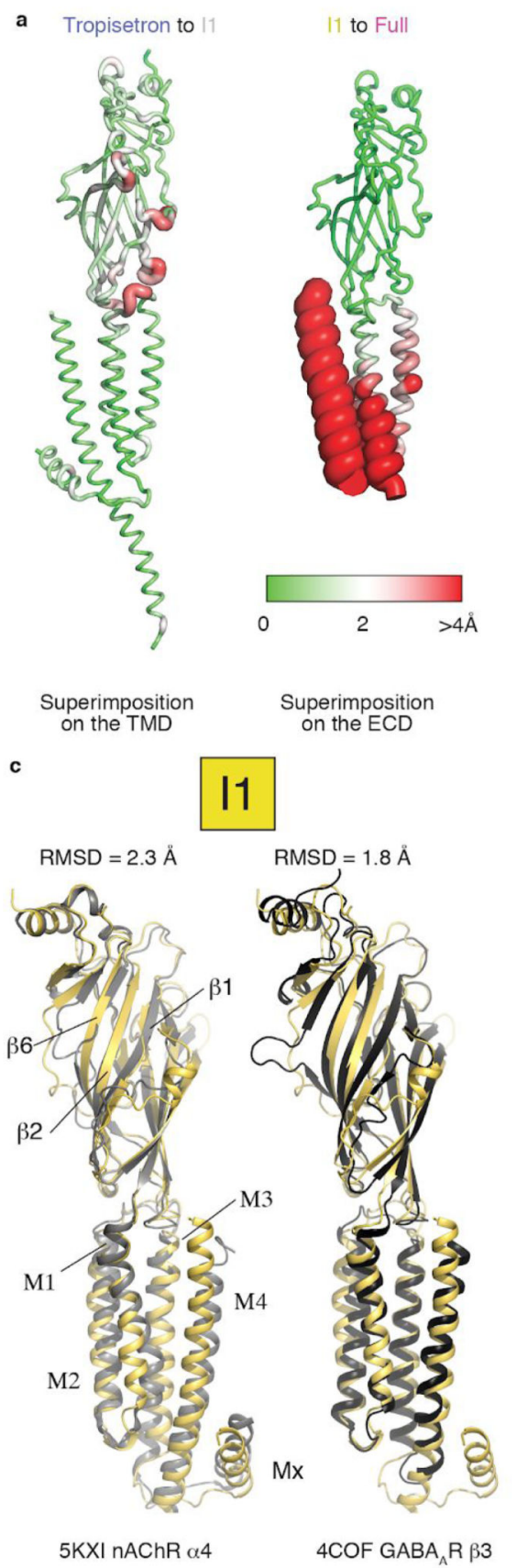

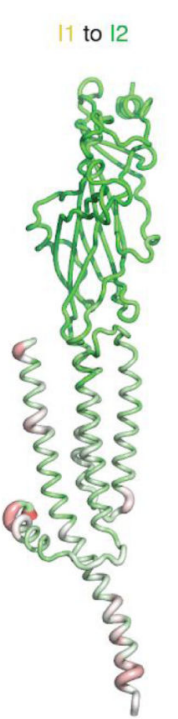

Superimposition on the ECD

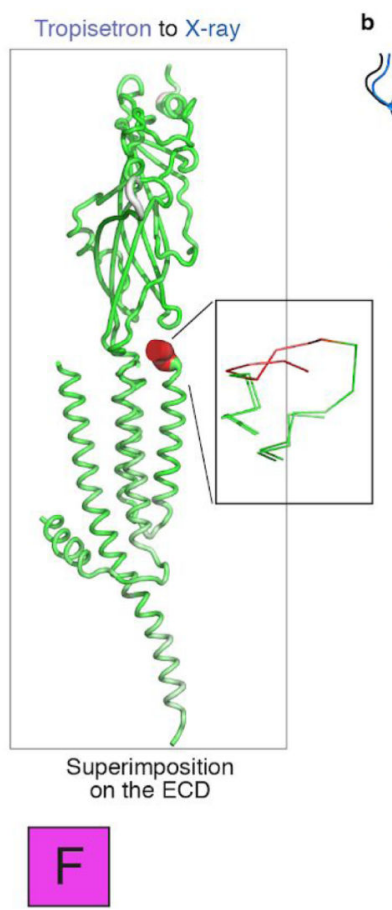

on the ECD
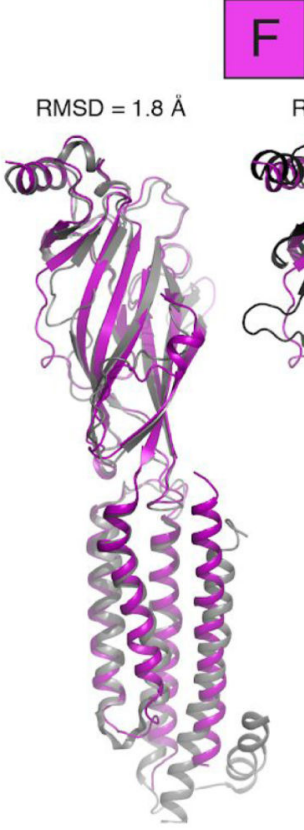

$5 \mathrm{KXI}$ nAChR $\alpha 4$
Tropisetron to

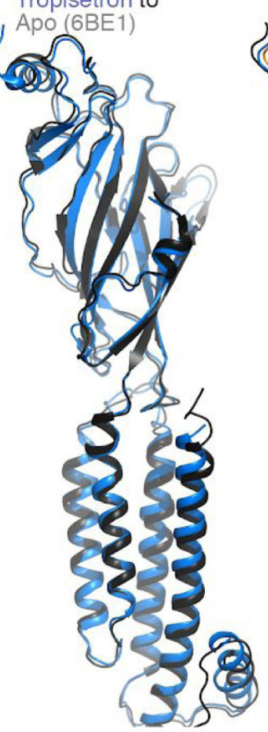

$\mathrm{RMSD}=1.15 \AA$

12
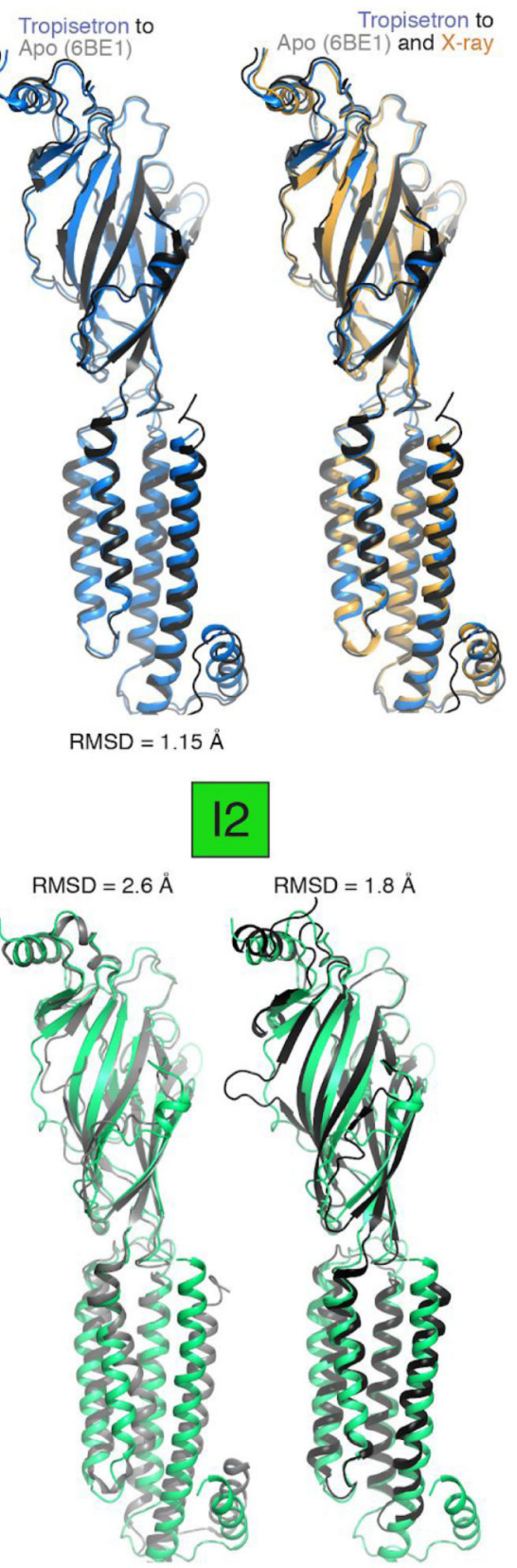

$5 \mathrm{KXI} n A C h R \alpha 4$

Extended Data Figure 7. Structural superimpositions with the $\mathrm{nAChR} a 4 \beta 2$, the $G A B A_{A} R \beta$, the apo 5-HT3 receptor structures

a. Putty representations of pairwise deviations for the 5-HT3 receptor conformations. The selection used for superimposition, and the two conformations used are noted for each image. The color code and tube thickness code are the same for all images. In the T versus $\mathrm{X}$-ray image, the red zone corresponds to a loop that was not modeled in the X-ray structure. b. Superimposition of a 5-HT3 receptor subunit in the inhibited T state (blue) and of a 5HT3 receptor subunit in the apo state (grey, PDB 6BE1). Structures are globally similar with differences in the lipid-exposed helices M3, Mx and M4, hypothetically a consequence of 
the different additives used - a lipid mixture in this study versus fluorinated fos-choline 8 for the apo structure. Superimposition of the T (blue), the apo structure (grey) and the X-ray structure (orange).

c. Superimposition of a 5-HT3 receptor subunit in the I1, I2, F conformations with a nAChR $a 4$ subunit (PDB 5KXI chain A) or a $\mathrm{GABA}_{\mathrm{A}} \mathrm{R} \beta 3$ subunit (PDB 4COF). $\mathrm{C} a$ RMSD are noted. Comparison of superimpositions with RMSD below $2 \AA$ shows that: in the case of F/ nAChR $a 4$ (panel a, purple/grey) the domain-to-domain orientation is very similar and the extracellular halves of helices M1, M2, M3 superimpose very well, while clear deviations are present in their intracellular halves and at the level of M4 (RMSD without M4 drops to 1.6 $\AA$ ). In the case of I1/GABAA $\beta 3$ (panel b, yellow/black) or I2/GABAA $\beta 3$ (panel b, green/black), differences are more distributed, deviations of the ECD indicate different domain-to-domain orientations, in the TMD M1 and M3 superimpose quite well but M2 and M4 clearly differ. 

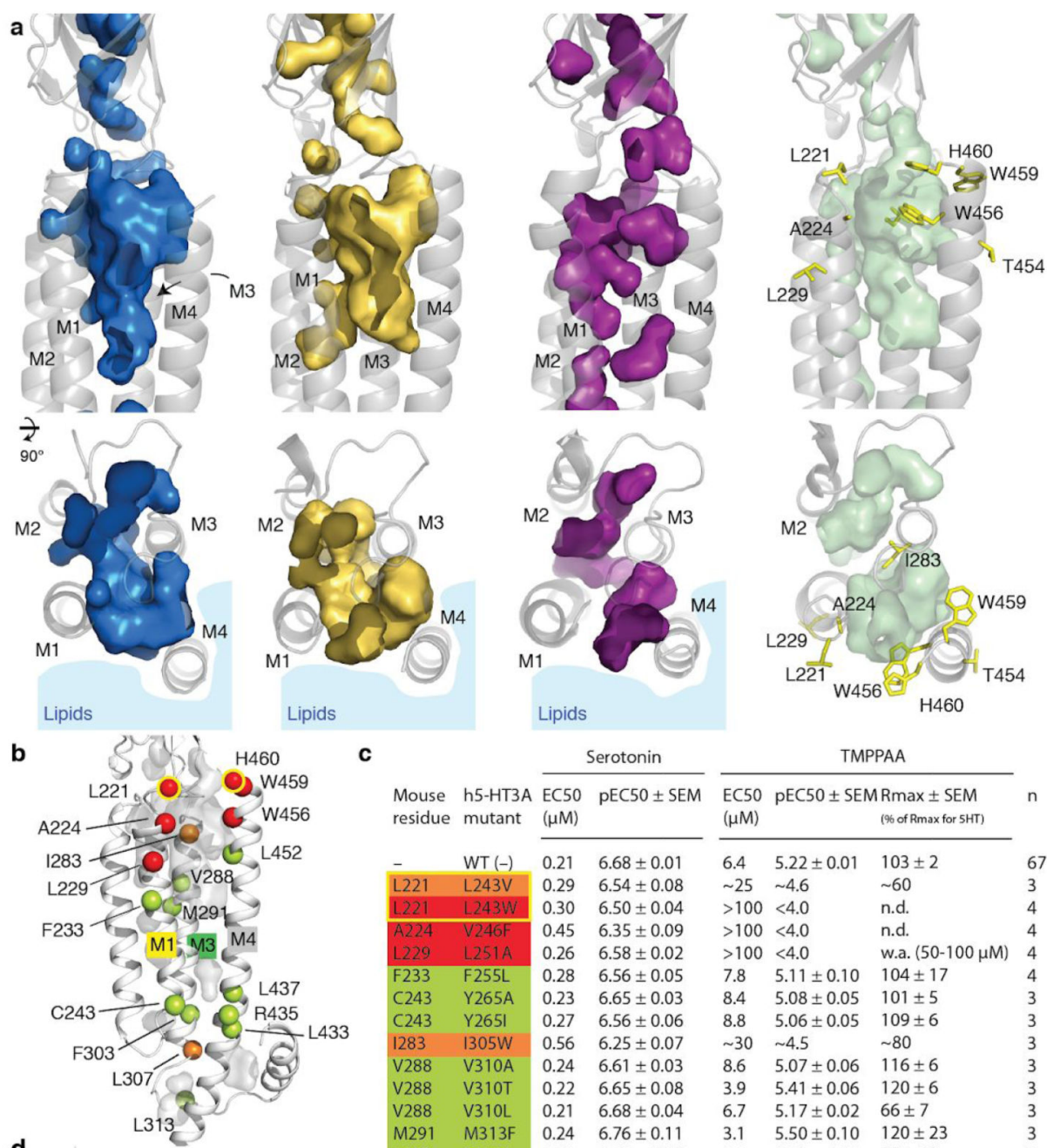

\begin{tabular}{llll} 
residue mutant & \multicolumn{2}{c}{$(\mu \mathrm{M})$} & \\
\cline { 3 - 4 }- & $\mathrm{WT}(-)$ & 0.21 & $6.68 \pm 0.01$ \\
\hline $\mathrm{L} 221$ & $\mathrm{~L} 243 \mathrm{~V}$ & 0.29 & $6.54 \pm 0.08$
\end{tabular}

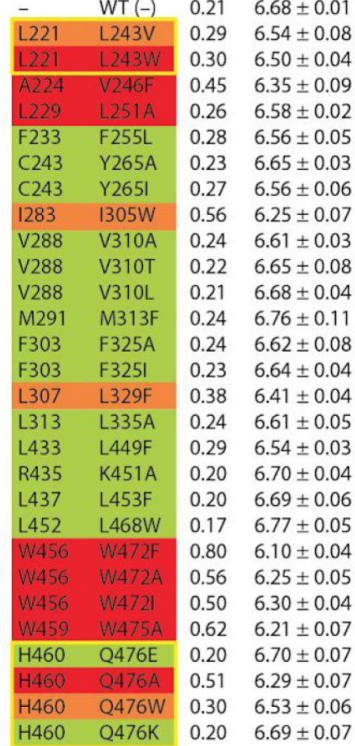

\begin{tabular}{|c|c|c|c|}
\hline & & AAA & \\
\hline $\begin{array}{l}\text { EC50 } \\
(\mu M)\end{array}$ & $\mathrm{pEC50} \pm$ SEN & $\begin{array}{l}\mathrm{MRmax} \pm \mathrm{SEM} \\
(\% \text { of Rmax for } 5 \mathrm{HT})\end{array}$ & $n$ \\
\hline 6.4 & $5.22 \pm 0.01$ & $103 \pm 2$ & \\
\hline$\sim 25$ & $\sim 4.6$ & $\sim 60$ & 3 \\
\hline$>100$ & $<4.0$ & n.d. & 4 \\
\hline$>100$ & $<4.0$ & n.d. & 4 \\
\hline$>100$ & $<4.0$ & w.a. $(50-100 \mu \mathrm{M})$ & 4 \\
\hline 7.8 & $5.11 \pm 0.10$ & $104 \pm 17$ & 4 \\
\hline 8.4 & $5.08 \pm 0.05$ & $101 \pm 5$ & 3 \\
\hline 8.8 & $5.06 \pm 0.05$ & $109 \pm 6$ & 3 \\
\hline$\sim 30$ & $\sim 4.5$ & $\sim 80$ & 3 \\
\hline 8.6 & $5.07 \pm 0.06$ & $116 \pm 6$ & 3 \\
\hline 3.9 & $5.41 \pm 0.06$ & $120 \pm 6$ & 3 \\
\hline 6.7 & $5.17 \pm 0.02$ & $66 \pm 7$ & 3 \\
\hline 3.1 & $5.50 \pm 0.10$ & $120 \pm 23$ & 3 \\
\hline 5.1 & $5.29 \pm 0.16$ & $95 \pm 10$ & \\
\hline 8.3 & $5.08 \pm 0.04$ & $99 \pm 7$ & \\
\hline$\sim 30$ & $\sim 4.5$ & $\sim 100$ & 3 \\
\hline 8.8 & $5.05 \pm 0.04$ & $69 \pm 11$ & 3 \\
\hline 8.2 & $5.08 \pm 0.05$ & $92 \pm 6$ & 3 \\
\hline 5.4 & $5.27 \pm 0.02$ & $101 \pm 5$ & 3 \\
\hline 8.0 & $5.09 \pm 0.09$ & $81 \pm 4$ & \\
\hline 7.1 & $5.14 \pm 0.05$ & $101 \pm 10$ & \\
\hline$>100$ & $<4.0$ & n.d. & \\
\hline$>100$ & $<4.0$ & n.d. & \\
\hline$>100$ & $<4.0$ & n.d. & 4 \\
\hline$>100$ & $<4.0$ & n.d. & 4 \\
\hline 4.0 & $5.39 \pm 0.03$ & $78 \pm 7$ & 4 \\
\hline$>100$ & $<4.0$ & w.a. $(50-100 \mu \mathrm{M})$ & \\
\hline$\sim 20$ & $\sim 4.7$ & $\sim 110$ & \\
\hline 7.7 & $5.11 \pm 0.10$ & $84 \pm 8$ & \\
\hline
\end{tabular}

Extended Data Figure 8. TMPPAA binds to an allosteric site in the TMD

a. Cavities in surface mode for the T (blue), I1 (yellow), F (purple) and I2 (green) states. Note the cavities re-arrangement from one state to any other. The protein is depicted as cartoon, with sticks on the right panels for residues where mutations impair the effect of TMPPAA. Noisy densities are found in the $\mathrm{I} 2$ reconstruction, in or close to the cavities, but they do not permit ligand modelling and could correspond to parts of a lipid or detergent molecule. 
b. Side view of the transmembrane domain of I 2 with mutated positions depicted as green spheres (for residues where mutations had negligible effect on TMPPAA potency), orange spheres (for residues where mutations reduced TMPPAA potency substantially) or red spheres (for mutations completely or almost completely eliminated TMPPAA activity at concentrations up to $100 \mu \mathrm{M}$ ). Yellow circles indicate residues where different mutations produced different effects.

c. Functional properties exhibited by serotonin and TMPPAA as agonists at $5-\mathrm{HT}_{3}$ receptors expressed in tsA201 cell in a fluorescence-based membrane potential (FMP) assay. The human $5-\mathrm{HT}_{3} \mathrm{~A}$ receptor was used for these experiments, since TMPPAA evokes a more robust agonist response through this receptor than through mouse $5-\mathrm{HT}_{3} \mathrm{~A}$ in this assay ${ }^{17}$. The color code is similar to that of panel b. n.d., not determinable, w.a., weak activity. A complete table of data for all tested mutant receptors is given in Table S5.

d. Concentration-response curves for serotonin (closed symbols) and TMPPAA (open symbols) tested as agonists at WT, W472A and L243W human 5-HT3A receptors expressed in tsA201 cells in the FMP assay. Data are from a single representative experiment determined at the same 96-well plate and are given as mean based on duplicate determinations. The experiment was repeated independently with similar results a total of 3 times for the W472A and L243W receptors and a total of 66 times for the WT receptor (n numbers for WT and all mutant receptors are given in Fig. 7c)

e. Loss of TMPPAA potency at the mouse W456A mutant receptor. Currents evoked by 10 $\mu \mathrm{M}$ serotonin (blue curve) or $10 \mu \mathrm{M}$ serotonin plus $100 \mu \mathrm{M}$ TMPPAA (red curve) are equivalent. The grey trace, obtained in an oocyte expressing WT receptors (response to 10 $\mu \mathrm{M}$ serotonin, normalized for the peak amplitude) shows that desensitization in WT and W456A mutant receptors are similar. Current traces are representative of 3 independently repeated experiments. 


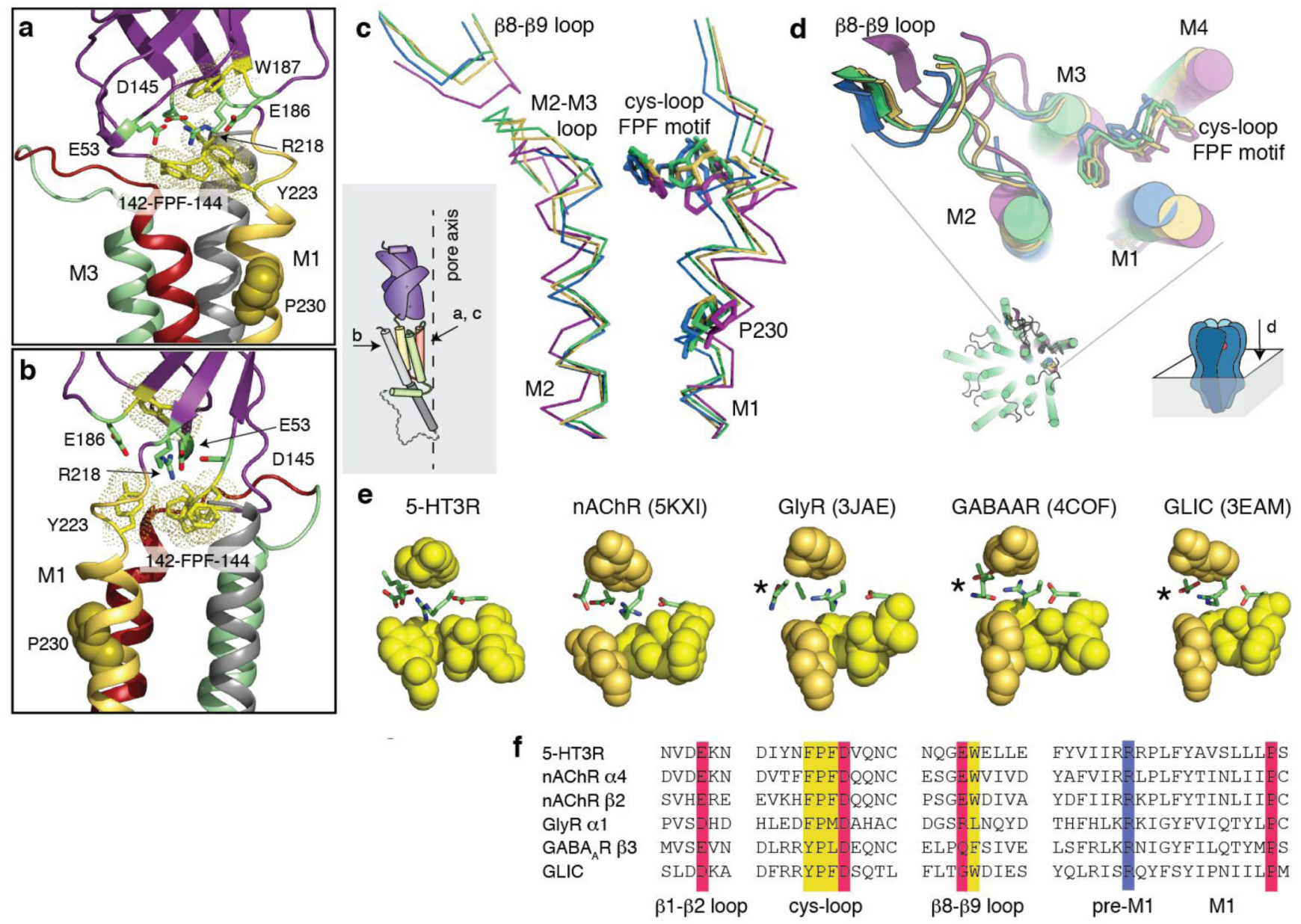

Extended Data Figure 9. Structural motif and reorganisation at the ECD-TMD interface a and b. Key interacting charged residues R218 (pre-M1, key in gating ${ }^{72-74}$ ), E53 (loop $\beta 1$ $\beta 2$ ), E186 (loop $\beta 8-\beta 9$, conserved only in cationic eukaryotic receptors), D145 (cys-loop) represented as purple sticks, are sandwiched between conserved essential aromatic residues (FPF motif of the cys-loop, W187 of $\beta 8-\beta 9$, Y223 of M1) represented as yellow sticks and dots. Lower in the membrane, the strictly conserved P230 represented as spheres allows M1 to kink. This structural organization is common to pLGIC of known structure. Orientation of the views are indicated on the topology scheme.

c and d. Side and top views depicting the concerted relative positions of the cys-loop FPF 'wedge' represented as sticks, of transmembrane helices, and of the $\beta 8$ - $\beta 9$ loop. Compared to the inhibited structure, the wedge moves towards and pushes on M1, and also moves towards M4 in I1, I2 and F. F presents the biggest re-organization in this zone: M1, the M2M3 loop, M3 follow the motion of the FPF motif while M2 is allowed to move away from the pore axis (that is, to the back in the view of panel $\mathrm{c}$ and to the top in panel $\mathrm{d}$ ).

e. Conservation of the 'sandwich' structural motif in representative pLGIC structures. The aromatic top and bottom layers are here represented as spheres (yellow: cys-loop, orange: $\beta 8-\beta 9$ loop and pre-M1 residue) while the central layer of charges is depicted as sticks. Note that in anionic receptors and GLIC, the charge of $\beta 8-\beta 9$ is not there (noted with a star). 
f. Sequence alignment of the motif residues in representative pLGIC structures. 

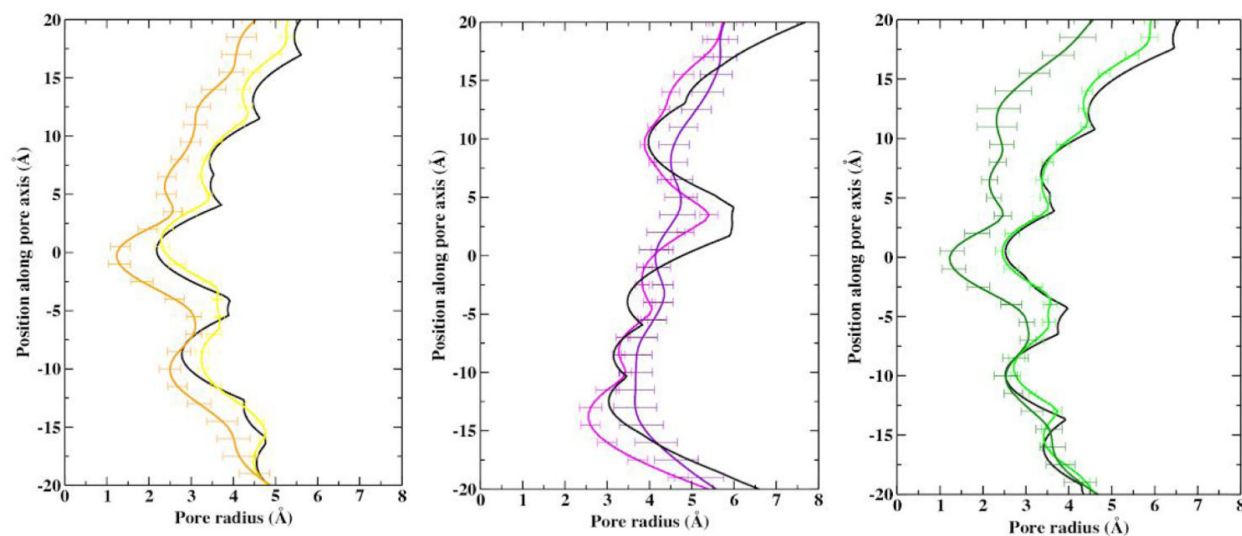

11

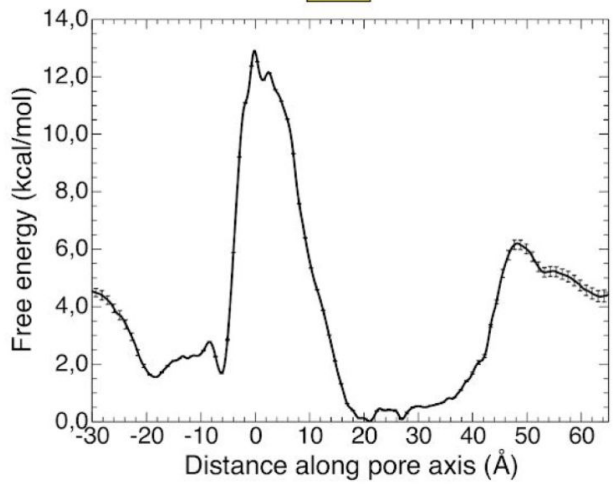

d X-ray

11

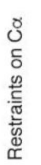
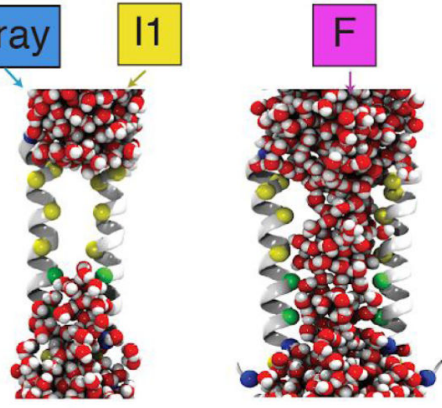

$\sqrt{2}+2$

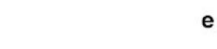

e
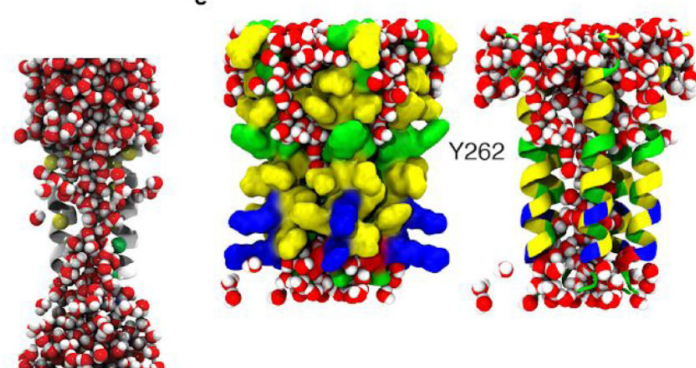

12

Extended Data Figure 10. Geometry of the pore during the molecular dynamics simulations a. HOLE transmembrane pore profiles for the trajectories starting from I1, F and I2 are depicted. In each graph, the cryo-EM structure profile is represented in black, the average profile during the restrained part of the simulation (30-60 ns, mean of $n=30$ snapshots taken every ns with S.D.) in light color, the average profile during the unrestrained part (60-1000 ns, mean of $n=940$ snapshots taken every ns with S.D.) in dark color. The $y$-axis origin correspond to L260/9' while S253/2's sits at about -12 A.

Nature. Author manuscript; available in PMC 2019 July 08. 
b. Complete potentials of mean force of $\mathrm{K}^{+}$ions as a function of position along the pore axis. The inset on the right side represents the $2 \mathrm{D}$ free-energy landscape as a function of the position along the pore axis and the radial direction, orthogonal to it. The represented freeenergy profile is the mean of $135,000,000$ values distributed along $2.16 \mu$ s of the I1 trajectory $(190,000,000$ values distributed along $3.04 \mu$ s of the F trajectory); the standard deviation corresponds in both cases to 50,000,000 values from four independent walkers, distributed along the last $0.8 \mu$ s of the trajectory.

c. Representative snapshots of the pore wetting during the initial restrained part (30-60 ns) of the simulations. A dewetted pore is observed for the T and I1 trajectories, a fully wetted one for the F trajectory, while wetting and dewetting occur in the I2 trajectory. $\mathrm{C} a$ atoms of pore-exposed residues are represented as spheres.

d. Snapshot of the I2 trajectory depicting the wetting of grooves at the back of M2 (shown as molecular surface on the left, as carton on the right) concomitant with wetting of the pore. 


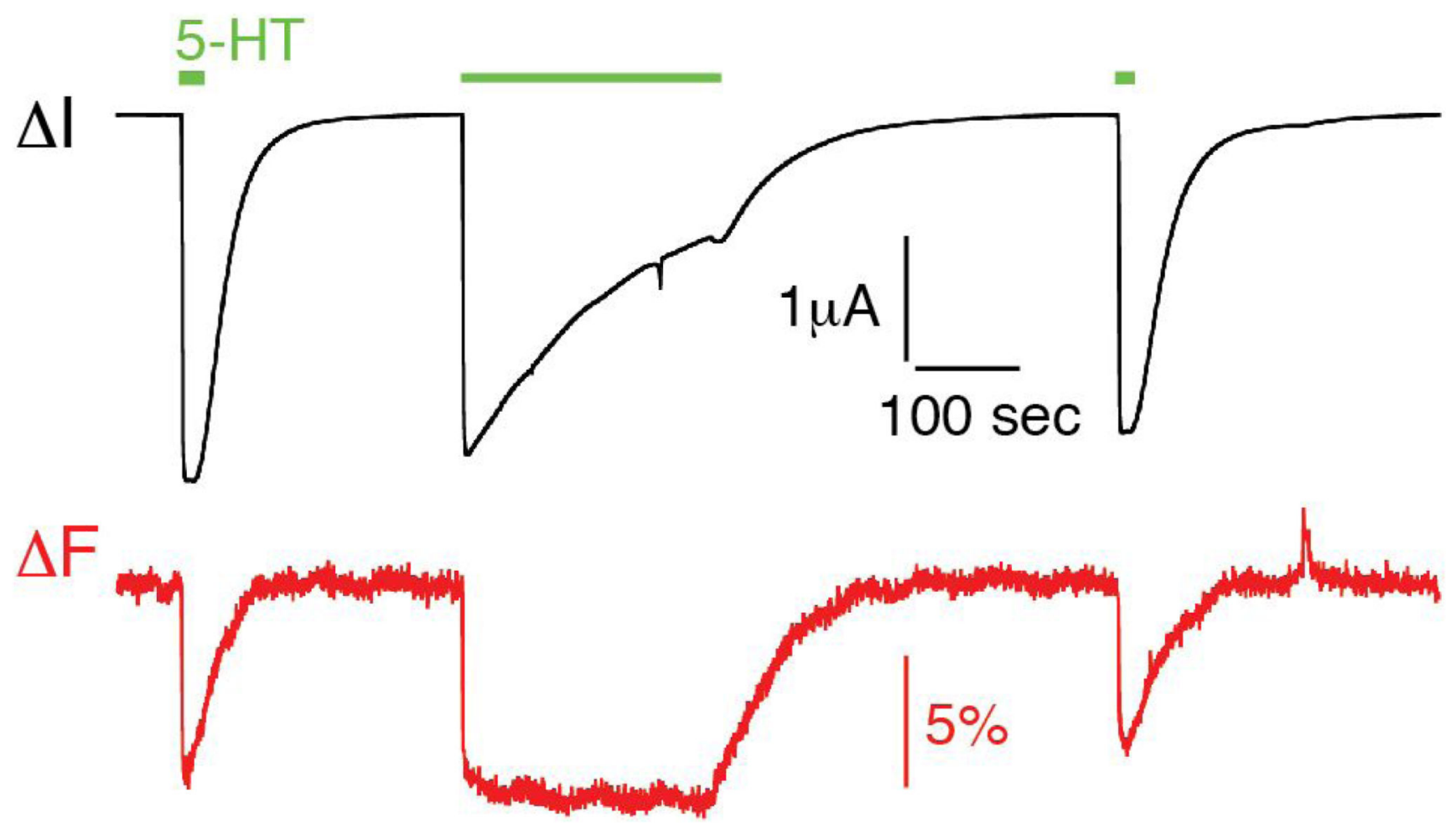

Extended Data Figure 11. Local motions in the pore probed by Voltage-clamp fluorometry Representative serotonin-evoked simultaneous current and fluorescence recordings from oocytes expressing S19'C receptors labeled with MTS-TAMRA. Similar traces were obtained in 3 independent experiments.

\section{Supplementary Material}

Refer to Web version on PubMed Central for supplementary material.

\section{Acknowledgments}

We acknowledge access to the C-CINA and ESRF Krios microscopes, and thank M. Chami, L. Kovacik and H. Stahlberg, G. Effantin, E. Kandiah and M. Hons for wonderful support. We are grateful to L. Estrozi, M. Bacia, G. Effantin, A. Desfosses for advice with cryo-EM, to the Vivaudou and Moreau laboratories for providing highquality oocytes and helping in electrophysiology, to members of the Nury laboratory, M. Gielen, E. Pebay-Peyroula for discussions.

The work was funded by the Marie Curie CIG NeuroPenta, the ERC Starting grant 637733 (H.N.). It used the platforms of the Grenoble Instruct-ERIC Center (ISBG: UMS 3518 CNRS-CEA-UGA-EMBL) with support from FRISBI (ANR-10 INSB-05-02) and GRAL (ANR-10-LABX-49-01) within the Grenoble PSB. The EM facility is supported by the Rhône-Alpes Region, the FRM, the FEDER and the GIS-IBISA.

\section{References}

1. Thompson AJ, Lester HA, Lummis SCR. The structural basis of function in Cys-loop receptors. Q Rev Biophys. 2010; 43:449-499. [PubMed: 20849671]

2. Nemecz À, Prevost MS, Menny A, Corringer P-J. Emerging Molecular Mechanisms of Signal Transduction in Pentameric Ligand-Gated Ion Channels. Neuron. 2016; 90:452-470. [PubMed: 27151638] 
3. Sparling BA, DiMauro EF. Progress in the discovery of small molecule modulators of the Cys-loop superfamily receptors. Bioorg Med Chem Lett. 2017; 27:3207-3218. [PubMed: 28606760]

4. Sauguet L, et al. Crystal structures of a pentameric ligand-gated ion channel provide a mechanism for activation. Proceedings of the National Academy of Sciences. 2013

5. Hassaïne G, et al. X-ray structure of the mouse serotonin 5-HT3 receptor. Nature. 2014; 512:276281. [PubMed: 25119048]

6. Miller PS, Aricescu AR. Crystal structure of a human GABAA receptor. Nature. 2014; 512:270275. [PubMed: 24909990]

7. Du J, Lü W, Wu S, Cheng Y, Gouaux E. Glycine receptor mechanism elucidated by electron cryomicroscopy. Nature. 2015; 526:224-229. [PubMed: 26344198]

8. Morales-Perez CL, Noviello CM, Hibbs RE. X-ray structure of the human a4 $\beta 2$ nicotinic receptor. Nature. 2016; 538:411-415. [PubMed: 27698419]

9. Basak S, et al. Cryo-EM structure of 5-HT3A receptor in its resting conformation. Nat Commun. 2018; 9:514. [PubMed: 29410406]

10. Unwin N. Refined structure of the nicotinic acetylcholine receptor at 4A resolution. J Mol Biol. 2005; 346:967-989. [PubMed: 15701510]

11. Mukhtasimova N, Lee W, Wang H, Sine S. Detection and trapping of intermediate states priming nicotinic receptor channel opening. Nature. 2009

12. Lape R, Colquhoun D, Sivilotti LG. On the nature of partial agonism in the nicotinic receptor superfamily. Nature. 2008; 454:722-727. [PubMed: 18633353]

13. Menny A, et al. Identification of a pre-active conformation of a pentameric channel receptor. Elife. 2017; 6

14. Corradi J, Gumilar F, Bouzat C. Single-Channel Kinetic Analysis for Activation and Desensitization of Homomeric 5-HT3A Receptors. Biophys J. 2009; 97:1335-1345. [PubMed: 19720021]

15. Sakmann B, Patlak J, Neher E. Single acetylcholine-activated channels show burst-kinetics in presence of desensitizing concentrations of agonist. Nature. 1980; 286:71-73. [PubMed: 6248795]

16. Trick JL, et al. Functional Annotation of Ion Channel Structures by Molecular Simulation. Structure. 2016

17. Gasiorek A, et al. Delineation of the functional properties and the mechanism of action of TMPPAA, an allosteric agonist and positive allosteric modulator of 5-HT3 receptors. Biochem Pharmacol. 2016; 110-111:92-108.

18. Jin X, Steinbach JH. A Portable Site: A Binding Element for $17 \beta$-Estradiol Can Be Placed on Any Subunit of a Nicotinic $a 4 \beta 2$ Receptor. J Neurosci. 2011; 31:5045-5054. [PubMed: 21451042]

19. Mukhtasimova N, Sine SM. Nicotinic receptor transduction zone: invariant arginine couples to multiple electron-rich residues. Biophys J. 2013; 104:355-367. [PubMed: 23442857]

20. Cymes GD, Grosman C. The unanticipated complexity of the selectivity-filter glutamates of nicotinic receptors. Nat Chem Biol. 2012; 8:975-981. [PubMed: 23064317]

21. Zhu F, Hummer G. Drying transition in the hydrophobic gate of the GLIC channel blocks ion conduction. Biophys J. 2012; 103:219-227. [PubMed: 22853899]

22. Aryal P, Sansom MSP, Tucker SJ. Hydrophobic gating in ion channels. J Mol Biol. 2015; 427:121130. [PubMed: 25106689]

23. Gielen M, Thomas P, Smart TG. The desensitization gate of inhibitory Cys-loop receptors. Nat Commun. 2015; 6

24. McKinnon NK, Bali M, Akabas MH. Length and Amino Acid Sequence of Peptides Substituted for the 5-HT3A Receptor M3M4 Loop May Affect Channel Expression and Desensitization. PLoS One. 2012; 7:e35563. [PubMed: 22539982]

25. Pless SA, Dibas MI, Lester HA, Lynch JW. Conformational variability of the glycine receptor M2 domain in response to activation by different agonists. J Biol Chem. 2007; 282:36057-36067. [PubMed: 17911099]

26. Dahan DS, et al. A fluorophore attached to nicotinic acetylcholine receptor $\beta \mathrm{M} 2$ detects productive binding of agonist to the $a \delta$ site. Proc Natl Acad Sci U S A. 2004; 101:10195-10200. [PubMed: 15218096] 
27. daCosta CJB, Baenziger JE. Gating of pentameric ligand-gated ion channels: structural insights and ambiguities. Structure. 2013; 21:1271-1283. [PubMed: 23931140]

28. Gonzalez-Gutierrez G, Wang Y, Cymes GD, Tajkhorshid E, Grosman C. Chasing the open-state structure of pentameric ligand-gated ion channels. J Gen Physiol. 2017; 48

29. Hibbs RE, Gouaux E. Principles of activation and permeation in an anion-selective Cys-loop receptor. Nature. 2011; 474:54-60. [PubMed: 21572436]

30. Hassaïne G, et al. Large scale expression and purification of the mouse 5-HT3 receptor. Biochimica et Biophysica Acta (BBA) - Biomembranes. 2013; 1828:2544-2552. [PubMed: 23747684]

31. Hassaïne G, et al. Expression, Biochemistry, and Stabilization with Camel Antibodies of Membrane Proteins: Case Study of the Mouse 5-HT3 Receptor. Methods Mol Biol. 2017; 1635:139-168. [PubMed: 28755368]

32. Yakel JL, Lagrutta A, Adelman JP, North RA. Single amino acid substitution affects desensitization of the 5-hydroxytryptamine type 3 receptor expressed in Xenopus oocytes. Proc Natl Acad Sci U S A. 1993; 90:5030-5033. [PubMed: 8506347]

33. $\mathrm{Hu} \mathrm{X}-\mathrm{Q}$, Lovinger DM. Role of aspartate 298 in mouse 5-HT 3Areceptor gating and modulation by extracellular Ca 2+ J Physiol. 2005; 568:381-396. [PubMed: 16096341]

34. Biyani N, et al. Focus: The interface between data collection and data processing in cryo-EM. J Struct Biol. 2017; 198:124-133. [PubMed: 28344036]

35. Zheng SQ, et al. MotionCor2: anisotropic correction of beam-induced motion for improved cryoelectron microscopy. Nat Methods. 2017; 14:331-332. [PubMed: 28250466]

36. Zhang K. Gctf: Real-time CTF determination and correction. J Struct Biol. 2016; 193:1-12. [PubMed: 26592709]

37. Scheres SHW. RELION: implementation of a Bayesian approach to cryo-EM structure determination. J Struct Biol. 2012; 180:519-530. [PubMed: 23000701]

38. Terwilliger TC, Sobolev O, Afonine PV, Adams PD. Automated map sharpening by maximization of detail and connectivity. bioRxiv. 2018

39. Adams PD, et al. PHENIX: a comprehensive Python-based system for macromolecular structure solution. Acta Crystallogr D Biol Crystallogr. 2010; 66:213-221. [PubMed: 20124702]

40. Emsley P, Lohkamp B, Scott WG, Cowtan K. Features and development of Coot. Acta Crystallogr D Biol Crystallogr. 2010; 66:486-501. [PubMed: 20383002]

41. Smart OS, Neduvelil JG, Wang X, Wallace BA, Sansom MS. HOLE: a program for the analysis of the pore dimensions of ion channel structural models. J Mol Graph. 1996; 14:354-60-376. [PubMed: 9195488]

42. Winn MD, et al. Overview of the CCP4 suite and current developments. Acta Crystallogr D Biol Crystallogr. 2011; 67:235-242. [PubMed: 21460441]

43. Pettersen EF, et al. UCSF Chimera--a visualization system for exploratory research and analysis. $\mathrm{J}$ Comput Chem. 2004; 25:1605-1612. [PubMed: 15264254]

44. Jo S, Kim T, Iyer VG, Im W. CHARMM-GUI: a web-based graphical user interface for CHARMM. J Comput Chem. 2008; 29:1859-1865. [PubMed: 18351591]

45. Wu EL, et al. CHARMM-GUI Membrane Builder toward realistic biological membrane simulations. J Comput Chem. 2014; 35:1997-2004. [PubMed: 25130509]

46. MacKerell AD, et al. All-atom empirical potential for molecular modeling and dynamics studies of proteins. J Phys Chem B. 1998; 102:3586-3616. [PubMed: 24889800]

47. Klauda JB, et al. Update of the CHARMM all-atom additive force field for lipids: validation on six lipid types. J Phys Chem B. 2010; 114:7830-7843. [PubMed: 20496934]

48. Best RB, et al. Optimization of the Additive CHARMM All-Atom Protein Force Field Targeting Improved Sampling of the Backbone $\phi, \psi$ and Side-Chain $\chi 1$ and $\chi 2$ Dihedral Angles. J Chem Theory Comput. 2012; 8:3257-3273. [PubMed: 23341755]

49. Jorgensen WL, Chandrasekhar J, Madura JD, Impey RW, Klein ML. Comparison of simple potential functions for simulating liquid water. J Chem Phys. 1983; 79:926-935. 
50. Hopkins CW, Le Grand S, Walker RC, Roitberg AE. Long-Time-Step Molecular Dynamics through Hydrogen Mass Repartitioning. J Chem Theory Comput. 2015; 11:1864-1874. [PubMed: 26574392]

51. Phillips JC, et al. Scalable molecular dynamics with NAMD. J Comput Chem. 2005; 26:17811802. [PubMed: 16222654]

52. Feller SE, Zhang Y, Pastor RW, Brooks BR. Constant pressure molecular dynamics simulation: The Langevin piston method. J Chem Phys. 1995; 103:4613-4621.

53. Darden T, York D, Pedersen L. Particle mesh Ewald: An $N \cdot \log (\mathrm{N})$ method for Ewald sums in large systems. J Chem Phys. 1993; 98:10089-10092.

54. Tuckerman M, Berne BJ, Martyna GJ. Reversible multiple time scale molecular dynamics. J Chem Phys. 1992; 97:1990-2001.

55. Ryckaert J-P, Ciccotti G, Berendsen HJC. Numerical integration of the cartesian equations of motion of a system with constraints: molecular dynamics of n-alkanes. J Comput Phys. 1977; 23:327-341.

56. Andersen HC. Rattle: A 'velocity' version of the shake algorithm for molecular dynamics calculations. J Comput Phys. 1983; 52:24-34.

57. Miyamoto S, Kollman PA. Settle: An analytical version of the SHAKE and RATTLE algorithm for rigid water models. J Comput Chem. 1992; 13:952-962.

58. Humphrey W, Dalke A, Schulten K. VMD: visual molecular dynamics. J Mol Graph. 1996; 14:33-8-27-8. [PubMed: 8744570]

59. Comer J, Phillips JC, Schulten K, Chipot C. Multiple-Replica Strategies for Free-Energy Calculations in NAMD: Multiple-Walker Adaptive Biasing Force and Walker Selection Rules. J Chem Theory Comput. 2014; 10:5276-5285. [PubMed: 26583211]

60 . Comer J, et al. The adaptive biasing force method: everything you always wanted to know but were afraid to ask. J Phys Chem B. 2015; 119:1129-1151. [PubMed: 25247823]

61. Moreau CJ, Niescierowicz K, Caro LN, Revilloud J, Vivaudou M. Ion channel reporter for monitoring the activity of engineered GPCRs. Methods Enzymol. 2015; 556:425-454. [PubMed: 25857794]

62. Yang J. Ion permeation through 5-hydroxytryptamine-gated channels in neuroblastoma N18 cells. J Gen Physiol. 1990; 96:1177-1198. [PubMed: 2286832]

63. Trattnig SM, et al. Discovery of a novel allosteric modulator of 5-HT3 receptors: inhibition and potentiation of Cys-loop receptor signaling through a conserved transmembrane intersubunit site. J Biol Chem. 2012; 287:25241-25254. [PubMed: 22589534]

64. Suryanarayanan A, et al. The loop C region of the murine 5-HT3A receptor contributes to the differential actions of 5-hydroxytryptamine and m-chlorophenylbiguanide. Biochemistry. 2005; 44:9140-9149. [PubMed: 15966738]

65. Yan D, Schulte MK, Bloom KE, White MM. Structural features of the ligand-binding domain of the serotonin 5HT3 receptor. J Biol Chem. 1999; 274:5537-5541. [PubMed: 10026168]

66. Ruepp M-D, Wei H, Leuenberger M, Lochner M, Thompson AJ. The binding orientations of structurally-related ligands can differ; A cautionary note. Neuropharmacology. 2017; 119:48-61. [PubMed: 28137449]

67. Kesters D, et al. Structural basis of ligand recognition in 5-HT(3) receptors. EMBO Rep. 2013; 14:49-56. [PubMed: 23196367]

68. Hibbs RE, et al. Structural determinants for interaction of partial agonists with acetylcholine binding protein and neuronal $a 7$ nicotinic acetylcholine receptor. EMBO J. 2009; 28:3040-3051. [PubMed: 19696737]

69. Spurny R, et al. Pentameric ligand-gated ion channel ELIC is activated by GABA and modulated by benzodiazepines. Proceedings of the National Academy of Sciences. 2012; 109:E3028-34.

70. Delbart F, et al. An allosteric binding site of the $a 7$ nicotinic acetylcholine receptor revealed in a humanized acetylcholine binding protein. J Biol Chem. 2017; doi: 10.1074/jbc.M117.815316

71. Steward LJ, et al. Importance of Phenylalanine 107 in Agonist Recognition by the 5Hydroxytryptamine3A Receptor. Mol Pharmacol. 2000; 57:1249-1255. [PubMed: 10825397] 
72. Purohit $\mathrm{P}$, Auerbach A. Acetylcholine receptor gating at extracellular transmembrane domain interface: the 'pre-M1' linker. J Gen Physiol. 2007; 130:559-568. [PubMed: 18040058]

73. Lee WY, Sine SM. Principal pathway coupling agonist binding to channel gating in nicotinic receptors. Nature. 2005; 438:243-247. [PubMed: 16281039]

74. Hu X-Q, Zhang L, Stewart RR, Weight FF. Arginine 222 in the pre-transmembrane domain 1 of 5HT3A receptors links agonist binding to channel gating. J Biol Chem. 2003; 278:46583-46589. [PubMed: 12970351] 

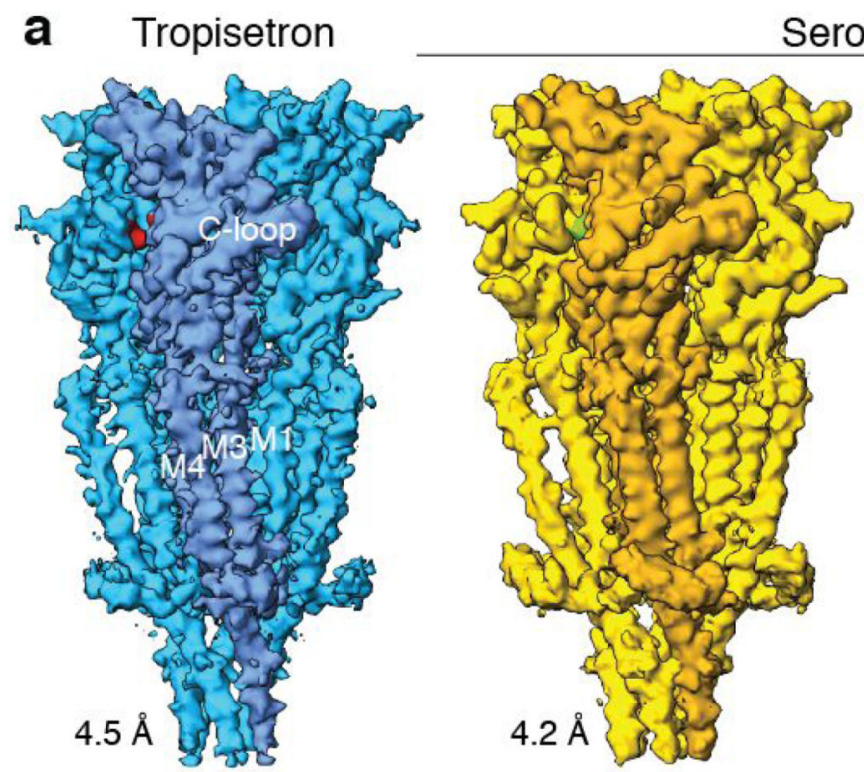

Serotonin
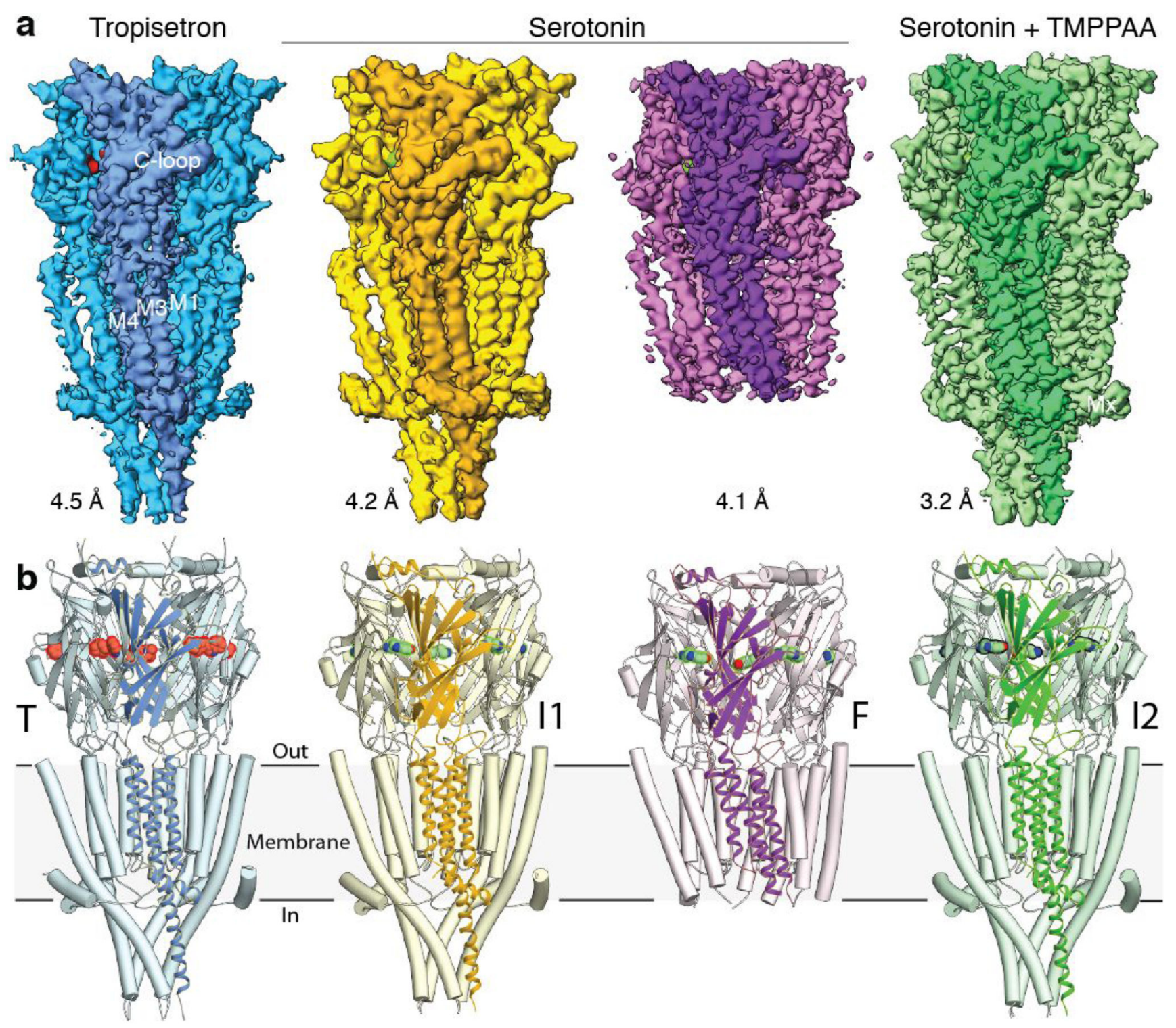

Figure 1. Homomeric 5-HT3A receptor 3D reconstructions and structures

a. Reconstructions and b. structures for the tropisetron dataset (protein in blue, ligand in red), the serotonin $+\mathrm{Ca}^{2+}$ dataset ( $\mathrm{I} 1$ in yellow and $\mathrm{F}$ in purple, ligand in green) and the serotonin + TMPPAA dataset (I2 in green, ligand in green). Resolutions according to the FSC 0.143 criterion are noted. 


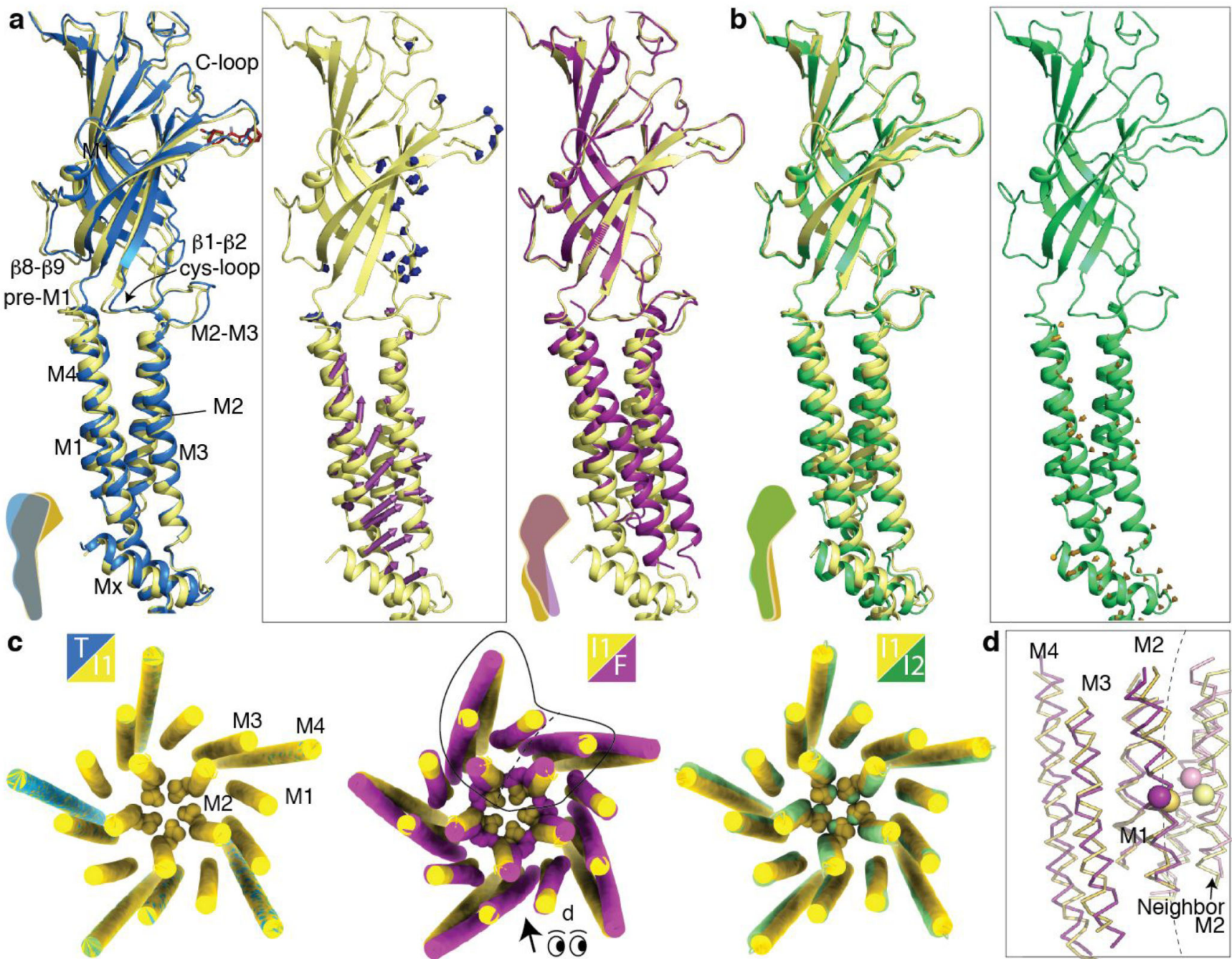

Figure 2. Transitions between the tropisetron-bound, the serotonin-bound and the serotonin +TMPPA-bound states

a. View parallel to the membrane of one subunit, for the serotonin-bound conformation I1 (yellow), overlaid with the tropisetron-bound conformation (blue, TMD superposition), with the serotonin-bound conformation F (purple, ECD superposition). In the inset, vectors indicate the local amplitude of motions, sampled on $\mathrm{C} a$ atoms $(\mathrm{T} \rightarrow \mathrm{I} 1$ in blue, $\mathrm{I} 1 \rightarrow \mathrm{F}$ in purple).

b. Overlay of the I1 (yellow) and the serotonin+TMPPAA-bound I2 (green) conformations; vector representations for the $\mathrm{I} 1 \rightarrow \mathrm{I} 2$ transition (ECD superposition).

c. Pairwise overlays of the TMD illustrating transitions at the quaternary level. Structures were superimposed on the ECD pentamer. L9' (L260) residues are shown as spheres. Line and arrow on the middle overlay indicate the region depicted in d. and the orientation of the view.

d.Tertiary reorganization within a subunit TMD. Overlay in ribbon representation of I2 and F with L260 Ca as spheres (TMD superposition within a single subunit). Note the vertical shift of M3-M4 relative to M1-M2, distortions on the extracellular halves of M1-M2, and 
interface re-arrangement with the neighbouring subunit helices (on the right of the dotted line). 


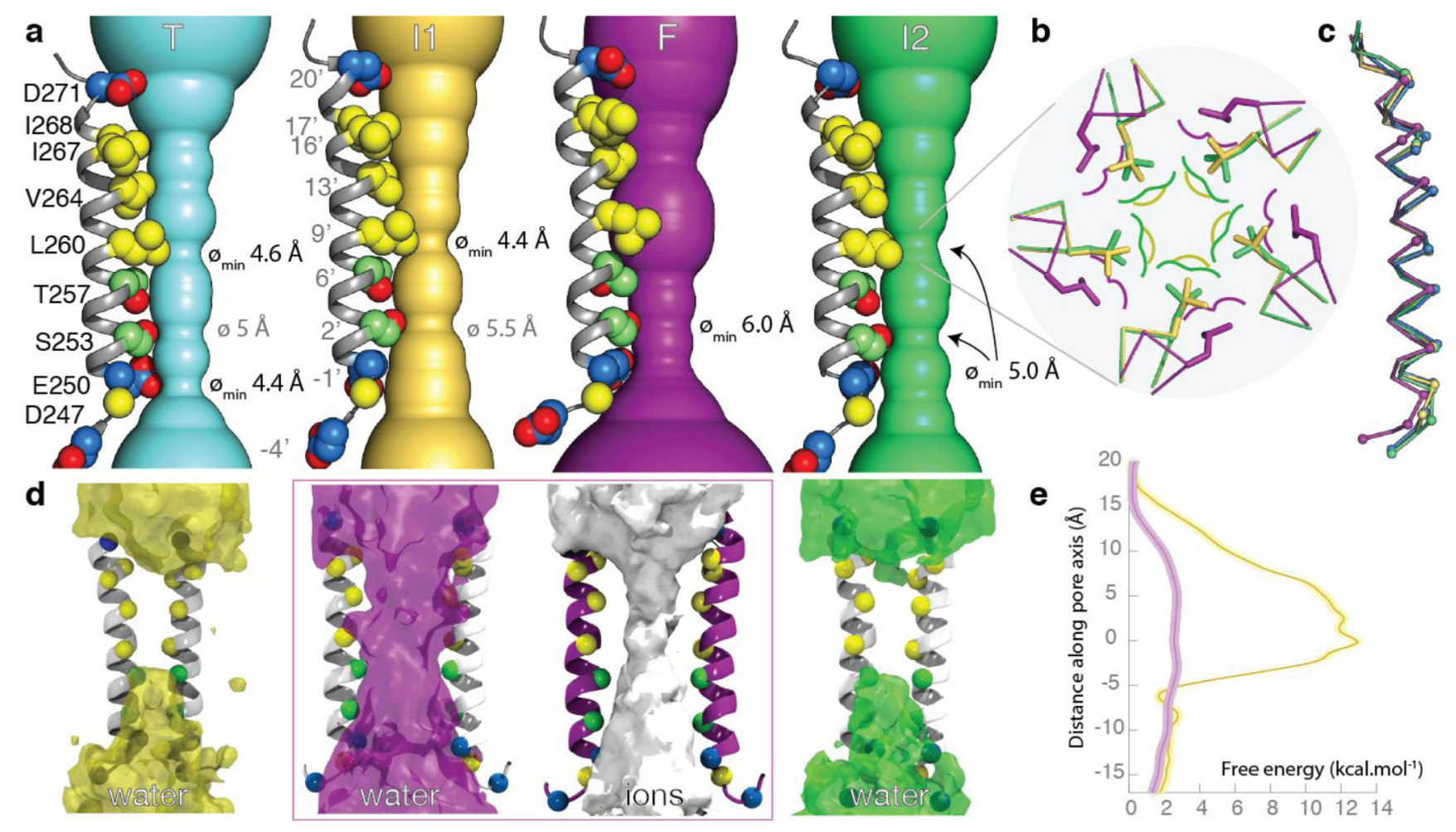

Figure 3. Geometry, wetting and energy landscape of the transmembrane pore

a. Static pore geometry: the accessible pathway through the pore is represented as a solid surface for each structure. Diameters of the constriction zones are noted. One M2 helix is depicted as cartoon with pore-exposed side chains as spheres (polar in green, hydrophobic in yellow, charged in blue). The view is parallel to the membrane plane.

b. Zoom on the hydrophobic gate constriction, formed at the level of L260 (sticks), which has a small motion backward in the $\mathrm{I} 2$ state and rotates out in the F state. Colored lines indicate molecular surfaces. The view is perpendicular to the membrane plane.

c. Superimposition of one M2 helix in the four conformations. Same view as in panel a.

d. Water densities of the pore region during the unrestrained part (100-1000 ns, panel e.) of simulations. Densities are depicted as transparent surfaces at the same contouring level. The density for $\mathrm{K}^{+}$ions is also included for the $\mathrm{F}$ trajectory featuring an open pore.

e. Potentials of mean force of $\mathrm{K}^{+}$ions as a function of the position along the pore axis. 


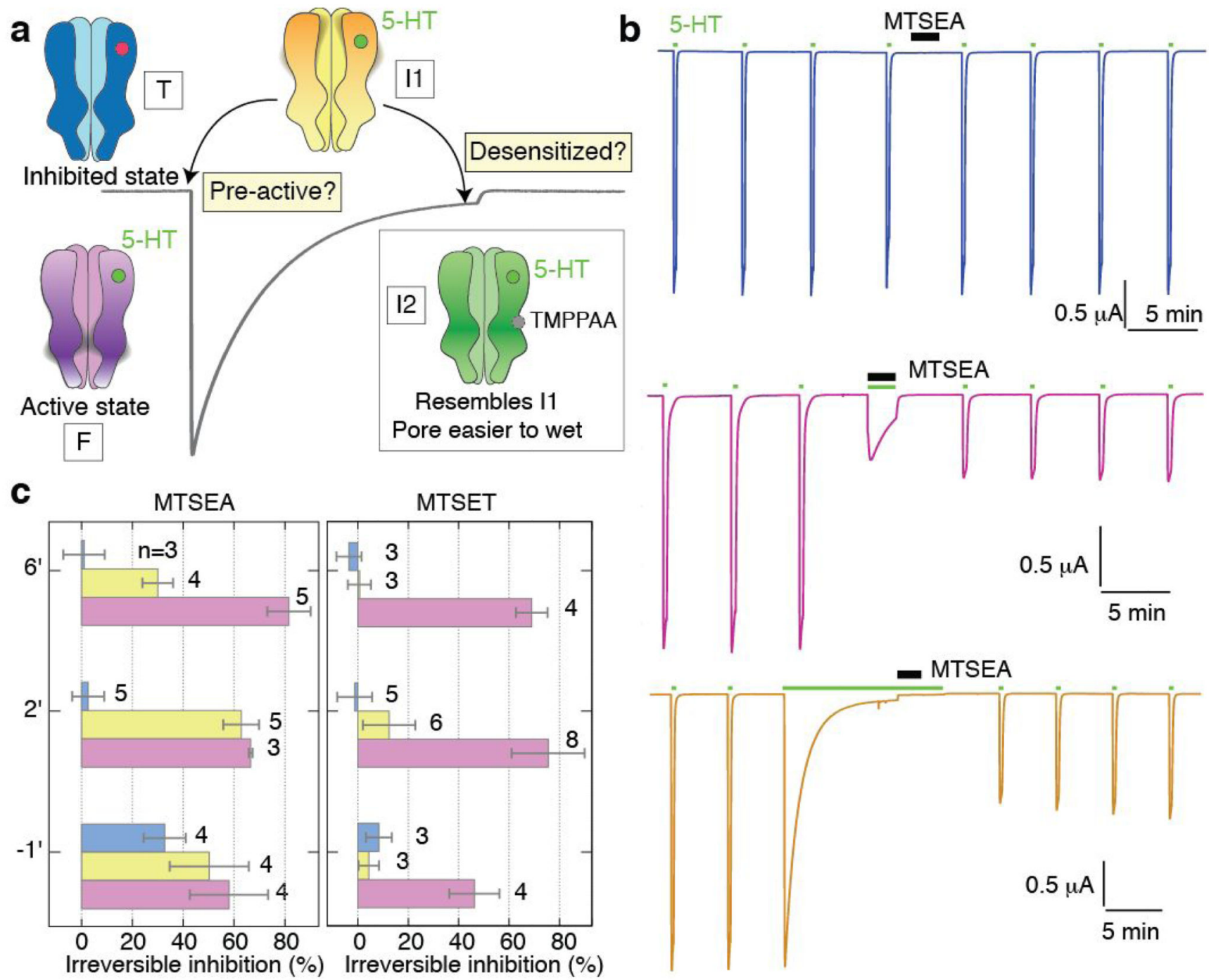

Figure 4. Putative molecular mechanisms of operation

a. T (blue) represents an inhibited state, stabilized by tropisetron (red disk). I1 (yellow) represents either a closed pre-active serotonin-bound (green disk) state or a desensitized state. F (purple) represents an open active state. The grey line illustrates the electrical response to serotonin recorded in an oocyte expressing 5-HT3 receptors.

b. Recordings of MTSEA ( $1 \mathrm{mM})$ modification on serotonin-evoked current in the mutant S2'C. MTSEA is applied in the resting (blue), the desensitized (orange), or active (purple) state. The protocol includes 10 -sec test serotonin applications before and after 2-min MTSEA modification.

c. Amount of current modification after MTSEA (1 mM) or MTSET (1 mM) modification, for mutants E-1'C, S2'C, T6'C in the resting (blue), the active (purple), or the desensitized (yellow) state. 